\title{
LOWER BOUNDS FOR MAXIMAL CP-RANKS OF COMPLETELY POSITIVE MATRICES AND TENSORS*
}

\author{
WERNER SCHACHINGER ${ }^{\dagger}$
}

\begin{abstract}
Let $p_{n}$ denote the maximal cp-rank attained by completely positive $n \times n$ matrices. Only lower and upper bounds for $p_{n}$ are known, when $n \geq 6$, but it is known that $p_{n}=\frac{n^{2}}{2}(1+o(1))$, and the difference of the current best upper and lower bounds for $p_{n}$ is of order $\mathcal{O}\left(n^{3 / 2}\right)$. In this paper, that gap is reduced to $\mathcal{O}(n \log \log n)$. To achieve this result, a sequence of generalized ranks of a given matrix $A$ has to be introduced. Properties of that sequence and its generating function are investigated. For suitable $\mathrm{A}$, the $d$ th term of that sequence is the cp-rank of some completely positive tensor of order $d$. This allows the derivation of asymptotically matching lower and upper bounds for the maximal cp-rank of completely positive tensors of order $d>2$ as well.
\end{abstract}

Key words. Completely positive matrices, Completely positive tensors, cp-rank, Copositive optimization.

AMS subject classifications. 15B48, 15A23, 15A03, 90C25.

1. Introduction and main results. In this paper, we consider completely positive matrices, as well as tensors of order greater than two, and their cp-rank. An $n \times n$ matrix $\mathrm{M}$ is said to be completely positive if for some $k \in \mathbb{N}$ there are vectors $\mathbf{v}_{1}, \ldots, \mathbf{v}_{k} \in \mathbb{R}_{+}^{n}$ such that there is a decomposition $\mathrm{M}=\sum_{i=1}^{k} \mathbf{v}_{i} \mathbf{v}_{i}^{\top}$, i.e., if $\mathrm{M}$ is in the convex hull $\mathcal{C} \mathcal{P}_{n}:=\operatorname{conv}\left\{\mathbf{x x}^{\top}: \mathbf{x} \in \mathbb{R}_{+}^{n}\right\}$. A completely positive matrix $\mathrm{M}$ usually has many such decompositions, and the $c p$-rank of $\mathrm{M}$, cpr $\mathrm{M}$, is the smallest $k$ allowing for such a decomposition. Similarly, we call a tensor $\mathrm{T} \in \mathcal{C} \mathcal{P}_{n, d}:=\operatorname{conv}\left\{\mathbf{x}^{\otimes d}: \mathbf{x} \in \mathbb{R}_{+}^{n}\right\}$ completely positive, and define cpr $\mathrm{T}$ to be the smallest $k$ such that $\mathbf{T}=\sum_{i=1}^{k} \mathbf{v}_{i}^{\otimes d}$ for some $\mathbf{v}_{1}, \ldots, \mathbf{v}_{k} \in \mathbb{R}_{+}^{n}$. Clearly, we have $\mathcal{C P} \mathcal{P}_{n, 2}=\mathcal{C} \mathcal{P}_{n}$. See the fundamental textbook [3] and the recent survey [2] on completely positive matrices, and [14, 24, 25] for early work on completely positive tensors for $d>2$, with the cp-rank of a tensor first appearing in [24]. Checking whether a tensor is completely positive is known to be NP-hard even in the $d=2$ case, see [13]. The set $\mathcal{C P}_{n}$ constitutes a proper cone (it is pointed, convex, closed, and has nonempty interior). With respect to the Frobenius inner product $\langle A, B\rangle:=\operatorname{trace}\left(A B^{\top}\right), \mathcal{C P}{ }_{n}$ is dual to the cone $\mathcal{C O P}{ }_{n}$ of symmetric copositive matrices of order $n$.

An $n \times n$ matrix $\mathrm{B}$ is said to be copositive if $\mathbf{x}^{\top} \mathrm{Bx} \geq 0$ for all $\mathbf{x} \in \mathbb{R}_{+}^{n}$. The two cones $\mathcal{C P}_{n}$ and $\mathcal{C O P}_{n}$ play an important role in the field of copositive optimization, which establishes a connection between discrete and continuous optimization, and has many real-world applications, see [6, 8, 12, 16]. See [22, 23, 29] for some recent uses of completely positive tensors in the field of optimization. From [23, Proposition 1], we know that $\mathcal{C P}_{n, d}$ is also a proper cone for $d \geq 3$. Furthermore, with respect to the inner product $\langle\mathrm{A}, \mathrm{B}\rangle:=(\operatorname{vec}(\mathrm{A}))^{\top} \operatorname{vec}(\mathrm{B})$, its dual cone, $\mathcal{C O} \mathcal{P}_{n, d}$, of symmetric copositive tensors, can be identified with the cone of $d$-degree copositive forms, i.e., homogeneous polynomials $P$ of degree $d$, satisfying $P(\mathbf{x}) \geq 0$ for $\mathbf{x} \in \mathbb{R}_{+}^{n}$. Here vec denotes vectorization of a tensor, so vec is a linear map from $\mathbb{R}^{n \times \cdots \times n}$ to $\mathbb{R}^{n^{d}}$, such that for $\mathbf{a}_{1}, \ldots, \mathbf{a}_{d} \in \mathbb{R}^{n}$ (by abuse of notation) the outer product $\mathbf{a}_{1} \otimes \cdots \otimes \mathbf{a}_{d}$ is mapped to the Kronecker

${ }^{*}$ Received by the editors on March 25, 2020. Accepted for publication on June 21, 2020. Handling Editor: Michael Tsatsomeros.

${ }^{\dagger}$ Department of Statistics and Operations Research (ISOR), University of Vienna, Vienna, Austria (werner.schachinger@univie.ac.at). 
product $\mathbf{a}_{1} \otimes \cdots \otimes \mathbf{a}_{d}$. The copositive form corresponding to $\mathrm{B} \in \mathcal{C O} \mathcal{P}_{n, d}$ is then $P_{\mathrm{B}}(\mathbf{x}):=\left\langle\mathrm{B}, \mathbf{x}^{\otimes d}\right\rangle$. We call $Z_{\mathrm{B}}=\left\{\mathbf{z} \in \Delta^{n}: P_{\mathrm{B}}(\mathbf{z})=0\right\}$ the zero set of $\mathrm{B}$, where $\Delta^{n}:=\left\{\mathbf{x} \in \mathbb{R}_{+}^{n}: \mathbf{x}^{\top} \boldsymbol{\eta}_{n}=1\right\}$ denotes the standard simplex, with $\boldsymbol{\eta}_{n}$ the all ones vector in $\mathbb{R}^{n}$. A tensor $\mathrm{B}$ is contained in the boundary of $\mathcal{C O} \mathcal{P}_{n, d}$ iff $Z_{\mathrm{B}}$ is nonempty. Tensors $\mathrm{A} \in \mathcal{C P}_{n, d}$ that are orthogonal to $\mathrm{B} \in \mathcal{C O} \mathcal{P}_{n, d}$ with finite zero set $Z_{\mathrm{B}}$, will play a central role in this paper.

Our main results establish improved lower bounds for

$$
p_{n}^{(d)}:=\max \left\{\operatorname{cpr} \boldsymbol{\top}: \mathrm{T} \in \mathcal{C} \mathcal{P}_{n, d}\right\},
$$

where for $d=2$, we denote $p_{n}^{(2)}$ by $p_{n}$. Currently, $p_{n}$ is only known for $1 \leq n \leq 5$, with $\left(p_{n}\right)_{1 \leq n \leq 5}=$ $(1,2,3,4,6)$, see [27]. For $n \geq 6$, only lower and upper bounds are available:

$$
p_{n} \leq s_{n}:=\left(\begin{array}{c}
n+1 \\
2
\end{array}\right)-4
$$

see [26], and

$$
p_{n} \geq s_{n}-\left\lfloor\frac{(n+5)(n-3)}{4}\right\rfloor=\left\lfloor\frac{n^{2}}{4}\right\rfloor, \quad \text { resp., } \quad p_{n} \geq s_{n}-n\left(\sqrt{2 n}-\frac{3}{2}\right),
$$

where the first lower bound is the DJL bound. It was conjectured by Drew, Johnson and Loewy [15] to be tight for $n \geq 4$, but is now known to be not tight for $n \geq 7$, see [9, 10]. Steps towards clarifying if it is tight for $n=6$ have been taken in [18,28]. The second lower bound appears in [7], where it is also observed that it is superior to the first one for $n \geq 15$, and follows from a bound given in [10, Theorem 2.2], where also some possible improvements are indicated, which however only affect the constant $\frac{3}{2}$. In this article we will show

$$
p_{n} \geq \frac{n^{2}}{2}-\left(2 \log _{2} \log n+e^{2}\right) n .
$$

We are only aware of a single result in the literature concerning $p_{n}^{(d)}$ with $d>2$ : In [17, Theorems 3.2 and $3.4]$ the case $n=2$ is considered and the inequality $p_{2}^{(d)} \leq 1+\left\lfloor\frac{d}{2}\right\rfloor$ shown. Furthermore, examples are given showing equality in the range $4 \leq d \leq 10$. Somewhat related, in [25, Corollary 3] it is shown that tensors in a certain subset of $\mathcal{C P}_{n, d}$ (the strongly symmetric, hierarchically dominated nonnegative tensors) have cp-ranks upper bounded by $\sum_{k=1}^{d}\left(\begin{array}{l}n \\ k\end{array}\right)$. In this article we will show a lower bound slightly stronger than

$$
p_{n}^{(d)} \geq\left(\begin{array}{l}
n \\
d
\end{array}\right)
$$

Our method of proof of these lower bounds is along the following lines: For given $\mathrm{B} \in \mathcal{C O} \mathcal{P}_{n, d}$ with $Z_{\mathrm{B}}$ finite, there exists $\mathrm{A} \in \mathcal{C} \mathcal{P}_{n, d}$ with cpr $\mathrm{A}$ equal to some number $\rho_{d}\left(Z_{\mathrm{B}}\right)$ computable from $Z_{\mathrm{B}}$. This number is regarded as being the $d$ th term of a sequence $\rho\left(Z_{\mathrm{B}}\right)=\left(\rho_{k}\left(Z_{\mathrm{B}}\right)\right)_{k \geq 0}$ of generalized ranks of a matrix with row set $Z_{\mathrm{B}}$. Being in need of $Z_{\mathrm{B}}$ with $\rho_{d}\left(Z_{\mathrm{B}}\right)$ large, we employ a construction that maps finite zero sets $\left(Z_{\mathrm{B}_{i}}\right)_{i=0}^{n_{0}}$ to some other finite zero set $Z_{\mathrm{B}}$, with $\mathrm{B}_{i} \in \mathcal{C O} \mathcal{P}_{n_{i}, d}$ for $0 \leq i \leq n_{0}$, and $\mathrm{B} \in \mathcal{C O} \mathcal{P}_{N, d}$, with $N=\sum_{i=1}^{n_{0}} n_{i}$, and succeed in expressing $\rho\left(Z_{\mathrm{B}}\right)$ in terms of $\left(\rho\left(Z_{\mathrm{B}_{i}}\right)\right)_{i=0}^{n_{0}}$. This constitutes a further major contribution of this paper.

The paper is organized as follows: In Section 2, we consider tensors $\mathrm{B}$ on the boundary of $\mathcal{C O} \mathcal{P}_{n, d}$, with $Z_{\mathrm{B}}$ finite. For $\mathrm{A} \in \mathcal{C P}_{n, d}$ such that $\mathrm{A} \perp \mathrm{B}$, there is a strong connection between the zeros of $\mathrm{B}$ and the cp-rank of $A$, that is dealt with in Corollary 2.2. Next, generalized ranks are introduced and studied 
in Propositions 2.4 and 2.5. In Section 3, we introduce generating polynomials for the sequence of rank differences. In Section 4, we recall the operation $\otimes$, that was introduced in [10], which turns a set of matrices relevant for our study into a semigroup. In Theorem 4.2, we show a homomorphism property: The generating polynomial of a product is the product of the generating polynomials. In Section 5, we introduce for any matrix $U$ a further operation, with as many operands (again matrices) as the number of columns of $\mathrm{U}$, and determine the corresponding generating polynomial in Theorem 5.3. In Section 6, and Section 7, we use the results from previous sections to derive lower bounds for $p_{n}^{(d)}$ in the cases $d>2$ and $d=2$.

In this paper, vectors are denoted by bold lowercase letters, and their components in the corresponding light letter. The zero vector and the $i$ th standard unit vector of appropriate dimension are denoted by $\mathbf{o}$ and $\mathbf{e}_{i}$, and $\mathrm{I}_{n}$ is the $n \times n$ identity matrix. Furthermore, we denote $[k: \ell]:=\{n \in \mathbb{Z}: k \leq n \leq \ell\}$, and rowsp A, colsp $A$, and coker $A$ denote the row space, the column space, and the cokernel of a matrix $A$.

2. Zero sets of copositive forms and generalized ranks. The following lemma, which essentially combines [9, Lemma 2.1], [10, Lemma 2.1], and Carathéodory's theorem (see [3]), will be used for inferring the existence of matrices, resp., tensors, of high cp-rank, and for deducing upper bounds for cp-ranks.

LEMma 2.1. Let $V$ be an Euclidian vector space. Assume that $U \subseteq V$ is such that $C:=$ cone $U$ is a proper cone. Denote by $C^{*}:=\{\mathbf{v} \in V:\langle\mathbf{u}, \mathbf{v}\rangle \geq 0$ for all $\mathbf{u} \in C\}$ its dual cone. Moreover, for a fixed $\mathbf{v} \in \partial C^{*}$, define $\mathcal{Z}(\mathbf{v}):=\{\mathbf{u} \in U:\langle\mathbf{v}, \mathbf{u}\rangle=0\}$, and let $r:=\operatorname{rank} \mathcal{Z}(\mathbf{v})$.

a) Assume $\mathbf{u} \in C$ with $\langle\mathbf{v}, \mathbf{u}\rangle=0$. Then for any representation $\mathbf{u}=\sum_{i=1}^{k} y_{i} \mathbf{u}_{i}$ with $\mathbf{y} \in \mathbb{R}_{+}^{k}$ and $\left\{\mathbf{u}_{1}, \ldots, \mathbf{u}_{k}\right\} \subseteq U$, we have $\left\{\mathbf{u}_{1}, \ldots, \mathbf{u}_{k}\right\} \subseteq \mathcal{Z}(\mathbf{v})$. For at least one such representation $k \leq r \leq \operatorname{dim} V$ holds.

b) If $\mathcal{Z}(\mathbf{v})$ is finite, then there exists $\mathbf{u} \in C$ such that $\mathbf{u}=\sum_{i=1}^{k} y_{i} \mathbf{u}_{i}$ with $\mathbf{y} \in \mathbb{R}_{+}^{k}$ and $\left\{\mathbf{u}_{1}, \ldots, \mathbf{u}_{k}\right\} \subseteq$ $\mathcal{Z}(\mathbf{v})$ implies $k \geq r$.

So, any vector in $C$ orthogonal to $\mathbf{v}$, when written as a nonnegative combination of vectors in $U$, exclusively uses vectors belonging to the set $\mathcal{Z}(\mathbf{v})$, there always being such a nonnegative combination that uses no more than $r$ vectors of $\mathcal{Z}(\mathbf{v})$. If $\mathcal{Z}(\mathbf{v})$ is finite, there is a vector in $C$ that uses at least $r$ vectors of $\mathcal{Z}(\mathbf{v})$, whenever written as a nonnegative combination of vectors in $U$. We will use the lemma with $V=\mathbb{R}^{n^{d}}$ and $U=\left\{\mathbf{x}^{\otimes d}: \mathbf{x} \in \Delta^{n}\right\}$. Note that $\left\langle\mathbf{x}^{\otimes d}, \boldsymbol{\eta}_{n^{d}}\right\rangle=1$ iff $\mathbf{x}^{\top} \boldsymbol{\eta}_{n}=1$. Clearly, we then have $C=\mathcal{C} \mathcal{P}_{n, d}$ and $C^{*}=\mathcal{C O} \mathcal{P}_{n, d}$, where for $\mathrm{B} \in \mathcal{C O} \mathcal{P}_{n, d}$, also $\mathcal{Z}(\mathrm{B})=\left\{\mathbf{z}^{\otimes d}: \mathbf{z} \in Z_{\mathrm{B}}\right\}$ holds. Noting that $\operatorname{dim} \mathcal{C} \mathcal{P}_{n, d}=\left(\begin{array}{c}n+d-1 \\ d\end{array}\right)$, Lemma 2.1 leads to the following corollary (see also [10, Lemma 2.2]):

Corollary 2.2. Let $\left\{\mathbf{u}_{1}, \ldots, \mathbf{u}_{k}\right\} \subseteq \mathbb{R}_{+}^{n}$ be the set of zeros of $\mathrm{B} \in \mathcal{C O} \mathcal{P}_{n, d}$. Then there exists $\mathbf{y} \in \mathbb{R}_{+}^{k}$ such that $\mathrm{A}:=\sum_{i=1}^{k} y_{i} \mathbf{u}_{i}^{\otimes d} \in \mathcal{C} \mathcal{P}_{n, d}$ satisfies $\operatorname{cpr} \mathrm{A}=\operatorname{rank}\left\{\mathbf{u}_{1}^{\otimes d}, \ldots, \mathbf{u}_{k}^{\otimes d}\right\}$. For any $\mathrm{A} \in \mathcal{C} \mathcal{P}_{n, d}$ we have cpr $\mathrm{A} \leq$ $\operatorname{dim} \mathcal{C} \mathcal{P}_{n, d}=\left(\begin{array}{c}n+d-1 \\ d\end{array}\right)$

For notational convenience (we want rows of certain matrices to correspond to tensors), we will be working with vectorized tensors, and thus, by $\otimes$ we henceforth denote the Kronecker product. We first generalize the notion of two-rank, introduced in [10]:

For a matrix $\mathrm{A}=\left[\mathbf{a}_{1}, \ldots, \mathbf{a}_{k}\right]^{\top} \in \mathbb{R}^{k \times n}$ we let $\mathrm{A}^{\langle 2\rangle}:=\left[\mathbf{a}_{1} \otimes \mathbf{a}_{1}, \ldots, \mathbf{a}_{k} \otimes \mathbf{a}_{k}\right]^{\top} \in \mathbb{R}^{k \times n^{2}}$, and more generally $\mathrm{A}^{\langle m\rangle}:=\left[\mathbf{a}_{1}^{\otimes m}, \ldots, \mathbf{a}_{k}^{\otimes m}\right]^{\top} \in \mathbb{R}^{k \times n^{m}}$, and define the $m$-rank of $\mathrm{A}$ as

$$
\operatorname{rank}^{(m)} \mathrm{A}:=\operatorname{rank} \mathrm{A}^{\langle m\rangle}
$$


In particular, we have $\mathrm{A}^{\langle 0\rangle}=\boldsymbol{\eta}_{k}$, and therefore, $\operatorname{rank}^{(0)} \mathrm{A}=1$ and $\operatorname{rank}^{(1)} \mathrm{A}=\operatorname{rank} \mathrm{A}$. Alternatively, $\operatorname{rank}^{(m)} \mathrm{A}=\operatorname{rank} \mathrm{G}^{(m)}$, where $\mathrm{G}^{(m)}$ is the $m$-th Hadamard (i.e., elementwise) power of the Gram matrix $\mathrm{G}=\mathrm{AA}^{\top}$ of the set of vectors $\left\{\mathbf{a}_{1}, \ldots, \mathbf{a}_{k}\right\}$.

EXAMPLE 2.3.

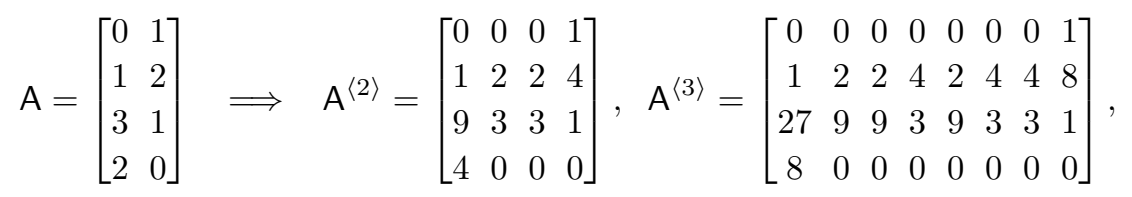

and therefore, $\operatorname{rank}^{(1)} \mathrm{A}=2, \operatorname{rank}^{(2)} \mathrm{A}=3$ and $\operatorname{rank}^{(3)} \mathrm{A}=4$.

We call a nonempty subset $U$ of a vector space $V$ pairwise linearly independent, iff either $U=\{\mathbf{v}\}$ with $\mathbf{v} \neq \mathbf{o}$, or $|U| \geq 2$ and $U$ does not contain two different vectors that are linearly dependent. Some fundamental observations concerning the sequence $\rho(\mathrm{A}):=\left(\operatorname{rank}^{(m)} \mathrm{A}\right)_{m \geq 0}$ are listed in the following propositions. Note that for the zero matrix $\mathrm{O} \in \mathbb{R}^{k \times n}$ we have $\rho(\mathrm{O})=(1,0,0, \ldots)$.

Proposition 2.4. Let $\mathrm{A}=\left[\mathbf{a}_{1}, \ldots, \mathbf{a}_{k}\right]^{\top} \in \mathbb{R}^{k \times n} \backslash\{\mathrm{O}\}$. Then for any submatrix $\mathrm{B}$ of $\mathrm{A}$ we have $\operatorname{rank}^{(m)} \mathrm{B} \leq \operatorname{rank}^{(m)} \mathrm{A}$ for all $m \geq 0$, which we denote $\rho(\mathrm{B}) \leq \rho(\mathrm{A})$. Moreover, $\rho\left(\mathrm{A}^{\prime}\right)=\rho(\mathrm{A})$ holds, if any of the following conditions is satisfied;

a) $\mathrm{A}^{\prime}=\mathrm{AQ}$, with $\mathrm{Q} \in \mathbb{R}^{n \times n}$ of full rank,

b) the columns of $\mathrm{A}^{\prime}$ consist of a maximal linearly independent subset of the column set of $\mathrm{A}$,

c) the rows of $\mathrm{A}^{\prime}$ consist of a maximal subset of pairwise linearly independent vectors of the row set of A (clearly, all such subsets are equal in size),

d) $\mathrm{A}^{\prime}=\mathrm{DPA}$, where $\mathrm{P} \in \mathbb{R}^{k \times k}$ is a permutation matrix and $\mathrm{D} \in \mathbb{R}^{k \times k}$ is a nonsingular diagonal matrix.

Proof. For the first assertion observe that $B$ being a submatrix of $A$ implies that $\mathrm{B}^{\langle m\rangle}$ is a submatrix of $A^{\langle m\rangle}$ for any $m \geq 0$. Regarding a), we have by well known properties of Kronecker products $(A Q)^{\langle m\rangle}=$ $\mathrm{A}^{\langle m\rangle} \mathrm{Q}^{\otimes m}$, with $\mathrm{Q}^{\otimes m} \in \mathbb{R}^{n^{m} \times n^{m}}$ of full rank. Therefore $\operatorname{rank}^{(m)} \mathrm{A}^{\prime}=\operatorname{rank}^{(m)} \mathrm{A}$. Turning to b) we first observe that removing $\mathbf{o}$ columns from $\mathbf{A}$ will not change $\rho(\mathbf{A})$. Then, by a), we may assume $\mathbf{A}=\left[\mathbf{b}_{1}, \ldots, \mathbf{b}_{n}\right]$ and $\mathrm{A}^{\prime}=\left[\mathbf{b}_{1}, \ldots, \mathbf{b}_{\ell}\right]$, with $\ell<n$ being the maximal cardinality of any linearly independent subset of the column set of $A$. Then there is $Q \in \mathbb{R}^{n \times n}$ of full rank, such that $A Q=\left[\mathbf{b}_{1}, \ldots, \mathbf{b}_{\ell}, \mathbf{o}, \ldots, \mathbf{o}\right]$, and thus, $\rho(\mathbf{A})=\rho(\mathbf{A Q})=\rho\left(\mathbf{A}^{\prime}\right)$, by a) and by removing $\mathbf{o}$ columns. Regarding $\mathrm{c}$ ), we can clearly remove any row consisting entirely of zeros from $\mathbf{A}$ without changing $\rho(\mathrm{A})$. If two nonzero rows $\mathbf{a}^{\top}$ and $\overline{\mathbf{a}}^{\top}$ of $\mathbf{A}$ are linearly dependent, then $\left(\mathbf{a}^{\otimes m}\right)^{\top}$ and $\left(\overline{\mathbf{a}}^{\otimes m}\right)^{\top}$ will be linearly dependent rows of $\mathbf{A}^{\langle m\rangle}$ for any $m \geq 0$. So, removing the row $\overline{\mathbf{a}}^{\top}$ from $\mathrm{A}$ will not change $\rho(\mathrm{A})$ either. For the proof of d), just note (PA $)^{\langle m\rangle}=\mathrm{PA}^{\langle m\rangle}$ and $(\mathrm{DA})^{\langle m\rangle}=\mathrm{D}^{m} \mathrm{~A}^{\langle m\rangle}$.

Proposition 2.5. Let $\mathrm{A} \in \mathbb{R}^{k \times n} \backslash\{\mathrm{O}\}$. Then the sequence $\rho(\mathrm{A})$ is eventually constant, that constant being the maximal number of pairwise linearly independent vectors of the row set of $\mathrm{A}$. Before reaching that constant, the sequence is strictly increasing.

Proof. By Proposition 2.4c), we may and do assume that the vectors in $\left\{\mathbf{a}_{1}, \ldots, \mathbf{a}_{k}\right\}$ are pairwise independent. Since $\mathrm{A}^{\langle m\rangle}$ has $k$ rows for all $m \geq 0, k$ is an upper bound for $\rho(\mathrm{A})$. By the assumed pairwise independence, there is a vector $\mathbf{u} \in \mathbb{R}^{n}$ such that $\mathbf{a}_{i}^{\top} \mathbf{u} \neq 0$ for all $i \in[1: k]$. By Proposition 2.4 d), we may and do assume that $\mathbf{a}_{i}^{\top} \mathbf{u}=1$ for all $i \in[1: k]$. Let now $\mathbf{v} \in \mathbb{R}^{n}$ be linearly independent of $\mathbf{u}$, such that $\left|\left\{\mathbf{a}_{i}^{\top} \mathbf{v}: i \in[1: k]\right\}\right|=k$, (such $\mathbf{v}$ exists by the assumed pairwise independence of the rows of $\mathrm{A}$ ) and find $\mathbf{q}_{i}, i \in[3: n]$, such that $\mathrm{Q}:=\left[\mathbf{u}, \mathbf{v}, \mathbf{q}_{3}, \ldots, \mathbf{q}_{n}\right]$ has full rank. Defining $\mathrm{A}^{\prime}:=\mathrm{AQ}$ and $\mathrm{A}_{2}:=\mathrm{A}[\mathbf{u}, \mathbf{v}]$, we have 
$\rho\left(\mathrm{A}_{2}\right) \leq \rho\left(\mathrm{A}^{\prime}\right)=\rho(\mathrm{A})$ by Proposition 2.4. The entries of the first column of $\mathrm{A}_{2}$ are all ones, those in the second column of $\mathrm{A}_{2}$ are all different, therefore some $k \times k$ submatrix of $\mathrm{A}_{2}^{\langle k\rangle}$ is a Vandermonde matrix with determinant $\prod_{1 \leq i<j \leq k}\left(\mathbf{a}_{j}^{\top} \mathbf{v}-\mathbf{a}_{i}^{\top} \mathbf{v}\right) \neq 0$. This shows $\operatorname{rank}^{(k)} \mathrm{A}_{2}=k$, so the upper bound $k$ is attained by $\rho(\mathrm{A})$. For what follows, we assume additionally w.l.o.g. that the first column of $A$, like that of $A^{\prime}$ before, consists of all ones. Then $A^{\langle m\rangle}$ is a submatrix of $A^{\langle m+1\rangle}$, and this implies containedness of left null spaces, i.e., coker $\mathrm{A}^{\langle m+1\rangle} \subseteq$ coker $\mathrm{A}^{\langle m\rangle}$, and thus, $\operatorname{rank}^{(m+1)} \mathrm{A} \geq \operatorname{rank}^{(m)} \mathrm{A}$, for any $m \geq 0$, which means that $\rho(\mathrm{A})$ is increasing (and eventually constant with value $k$ ). The assumption $\operatorname{rank}^{(m+1)} \mathrm{A}=\operatorname{rank}^{(m)} \mathrm{A}$ then implies coker $\mathrm{A}^{\langle m+1\rangle}=$ coker $\mathrm{A}^{\langle m\rangle}$, i.e., for some $n^{m} \times n^{m+1}$ matrix $\mathrm{Q}=\left(\mathrm{I}_{n^{m}} \mid \mathrm{R}\right)$ we have $\mathrm{A}^{\langle m+1\rangle}=\mathrm{A}^{\langle m\rangle} \mathrm{Q}$. Using in several places the mixed-product property $(A \otimes B)(C \otimes D)=A C \otimes B D$, we now observe

$$
\begin{aligned}
\mathrm{A}^{\langle m+2\rangle} & =\sum_{i=1}^{k} \mathbf{e}_{i}\left[\left(\mathbf{e}_{i}^{\top} \mathrm{A}^{\langle m+1\rangle}\right) \otimes\left(\mathbf{e}_{i}^{\top} \mathrm{A}\right)\right]=\sum_{i=1}^{k} \mathbf{e}_{i}\left(\mathbf{e}_{i}^{\top} \otimes \mathbf{e}_{i}^{\top}\right)\left(\mathrm{A}^{\langle m+1\rangle} \otimes \mathrm{A}\right) \\
& =\sum_{i=1}^{k} \mathbf{e}_{i}\left(\mathbf{e}_{i}^{\top} \otimes \mathbf{e}_{i}^{\top}\right)\left(\mathrm{A}^{\langle m\rangle} \otimes \mathrm{A}\right)\left(\mathrm{Q} \otimes \mathrm{I}_{n}\right)=\mathrm{A}^{\langle m+1\rangle}\left(\mathrm{Q} \otimes \mathrm{I}_{n}\right)
\end{aligned}
$$

showing coker $\mathrm{A}^{\langle m+2\rangle}=$ coker $\mathrm{A}^{\langle m+1\rangle}$. Thus, we obtain $\operatorname{rank}^{(m+2)} \mathrm{A}=\operatorname{rank}^{(m+1)} \mathrm{A}$, and induction yields $\operatorname{rank}^{(i)} \mathrm{A}=\operatorname{rank}^{(m)} \mathrm{A}$ for all $i>m$. We conclude that $\operatorname{rank}^{(m+1)} \mathrm{A}=\operatorname{rank}^{(m)} \mathrm{A} \operatorname{implies}^{\operatorname{rank}^{(m)}} \mathrm{A}=k, \operatorname{and}^{(m)}$ this completes the proof.

3. Generating polynomials and cyclically symmetric matrices. For matrices A we are now going to introduce the generating function (indeed a polynomial) of the sequence of differences of $\rho(\mathrm{A})$. This (and some proofs to come) is facilitated by the following definition.

Definition 3.1. Let the rows of $A \in \mathbb{R}^{k \times n}$ be pairwise linearly independent. We say that $A$ is rowadjusted (with $\ell+1$ blocks), if $\mathrm{A}=\left[\begin{array}{c}\mathrm{A}_{0} \\ \vdots \\ \mathrm{A}_{\ell}\end{array}\right]$, with $\mathrm{A}_{i} \in \mathbb{R}^{a_{i} \times n}, a_{i}>0$ for $i \in[0: \ell]$, and

$$
\operatorname{rank}^{(j)} \mathrm{A}=\operatorname{rank}^{(j)} \mathrm{A}_{[0: j]}=\sum_{i=0}^{j} a_{i}, \quad \text { for } j \in[0: \ell]
$$

where $\mathrm{A}_{[0: j]}:=\left[\begin{array}{c}\mathrm{A}_{0} \\ \vdots \\ \mathrm{A}_{j}\end{array}\right]$ for $j \in[0: \ell]$

It is easy to see that if $\mathrm{A}$ is row-adjusted, then the numbers $\ell$ and $\left(a_{i}\right)_{i \in[0: \ell]}$ are uniquely determined. If for some permutation matrix $P$ the matrix PA is also row-adjusted, then $A$ and PA share the number of blocks $\ell+1$ and the sequence of block sizes $\left(a_{i}\right)_{i \in[0: \ell]}$. Moreover, for any matrix $\mathrm{A} \in \mathbb{R}^{k \times n}$ with pairwise linearly independent rows (but not for $A=0$ ), there is a permutation matrix $P$ (in general not unique) such that PA is row-adjusted.

Definition 3.2. For $\mathrm{A} \in \mathbb{R}^{k \times n}$, we define its rank differences generating polynomial as

$$
p_{\mathrm{A}}(x)=1+\sum_{i \geq 0}\left(\operatorname{rank}^{(i+1)} \mathrm{A}-\operatorname{rank}^{(i)} \mathrm{A}\right) x^{i+1} .
$$

Clearly, $p_{\mathrm{A}}$ depends on $\mathrm{A}$ only via $\rho(\mathrm{A})$. If the rows of $\mathrm{A}$ are pairwise linearly independent, we may w.l.o.g. assume that $\mathrm{A}$ is row-adjusted, with block sizes $\left(a_{i}\right)_{i \in[0: \ell]}$, leading to the representation $p_{\mathrm{A}}(x)=$ $\sum_{i=0}^{\ell}\left(\operatorname{rank}^{(i)} \mathrm{A}_{i}\right) x^{i}=\sum_{i=0}^{\ell} a_{i} x^{i}$. 
EXAMPLE 3.3. Let

$$
A:=\left[\begin{array}{lll}
0 & 1 & 1 \\
1 & 2 & 0 \\
1 & 3 & 1 \\
0 & 4 & 0
\end{array}\right]
$$

Then $\left(\operatorname{rank}^{(i)} \mathrm{A}\right)_{i \in[0: 2]}=(1,3,4)$, and therefore $p_{\mathrm{A}}(x)=1+2 x+x^{2}$. The matrix $\mathrm{A}$ is not row-adjusted, since

$$
\operatorname{rank}\left[\begin{array}{ccc}
0 & 1 & 1 \\
1 & 2 & 0 \\
1 & 3 & 1
\end{array}\right]=2<3
$$

Swapping third and fourth row of $A$ yields the row-adjusted matrix $\left[\begin{array}{l}A_{0} \\ A_{1} \\ A_{2}\end{array}\right]$, where $A_{0}=\left[\begin{array}{lll}0 & 1 & 1\end{array}\right], A_{1}=\left[\begin{array}{lll}1 & 2 & 0 \\ 0 & 4 & 0\end{array}\right]$, and $\mathrm{A}_{2}=\left[\begin{array}{lll}1 & 3 & 1\end{array}\right]$.

The following corollary easily follows from the preceding propositions.

Corollary 3.4. Let $\mathrm{A} \in \mathbb{R}^{k \times n} \backslash\{\mathrm{O}\}$. Then $p_{\mathrm{A}}(x)$ is indeed a polynomial. All coefficients up to the leading one are positive. The rows of $\mathrm{A}$ are pairwise linearly independent iff $p_{\mathrm{A}}(1)=k$. If $\mathrm{D}, \mathrm{P}$ and $\mathrm{Q}$ are as in Proposition 2.4, then for $\mathrm{A}^{\prime}:=\mathrm{DPAQ}$, we have $p_{\mathrm{A}}(x)=p_{\mathrm{A}^{\prime}}(x)$.

We denote the support of a vector $\mathbf{v}=\left[v_{1}, \ldots, v_{n}\right]^{\top}$ by $I(\mathbf{v}):=\left\{i \in[1: n]: v_{i} \neq 0\right\}$. For $\mathbf{A}=\left[\mathbf{a}_{1}, \ldots, \mathbf{a}_{k}\right]^{\top} \in$ $\mathbb{R}^{k \times n}$ and $s \subseteq[1: n]$, we let $K_{s}(\mathrm{~A}):=\left\{j \in[1: k]: s \subseteq I\left(\mathbf{a}_{j}\right)\right\}$. Next, we let $\mathrm{A}_{s}:=\mathrm{A}_{K_{s}}(\mathbf{A}) \times[1: n]$ be the matrix whose rows are those rows of $\mathrm{A}$ that have supports containing $s$. In particular $\mathrm{A}_{\{\}}=\mathrm{A}$.

DeFInition 3.5. For $\mathrm{A} \in \mathbb{R}^{k \times n}$, we define its multivariate rank differences generating polynomial as

$$
p_{\mathrm{A}}^{\star}\left(x ; z_{1}, \ldots, z_{n}\right):=\sum_{s \subseteq[1: n]} p_{\mathrm{A}_{s}}(x) \prod_{i \in s}\left(z_{i}-1\right),
$$

where in case that $K_{s}(\mathrm{~A})=\{\}$ we define $p_{\mathrm{A}_{s}}$ to be the zero polynomial.

Obviously, $p_{\mathrm{A}}(x)=p_{\mathrm{A}}^{\star}(x ; 1, \ldots, 1)$ holds, and if $\mathrm{P}$ is an order $k$ permutation matrix and $\mathrm{D}_{i}, i \in\{1,2\}$, are nonsingular diagonal matrices of orders $k$ and $n$, then for $\mathrm{A}^{\prime}:=\mathrm{D}_{1} \operatorname{PAD}_{2}$, we have $p_{\mathrm{A}}^{\star}\left(x ; z_{1}, \ldots, z_{n}\right)=$ $p_{\mathrm{A}^{\prime}}^{\star}\left(x ; z_{1}, \ldots, z_{n}\right)$. If $\mathrm{P} \in \mathbb{R}^{n \times n}$ is a permutation matrix, then $p_{\mathrm{AP}}^{\star}\left(x ; z_{1}, \ldots, z_{n}\right)=p_{\mathrm{A}}^{\star}\left(x ; z_{\pi(1)}, \ldots, z_{\pi(n)}\right)$, where $\pi$ is defined by $\mathbf{e}_{i}^{\top} \mathrm{P}=\mathbf{e}_{\pi(i)}^{\top}$ for $i \in[1: n]$.

REMARK 3.6. a) Note that there is another representation of $p^{\star}$ :

$$
p_{\mathrm{A}}^{\star}\left(x ; z_{1}, \ldots, z_{n}\right)=\sum_{s \subseteq[1: n]} q_{\mathrm{A}, s}(x) \prod_{i \in s} z_{i},
$$

where $q_{\mathrm{A}, s}(x):=\sum_{s \subseteq t \subseteq[1: n]}(-1)^{|t|-|s|} p_{\mathrm{A}_{t}}(x)$, obtained by expanding the products $\prod_{i \in s}\left(z_{i}-1\right)$ and interchanging the order of summation.

b) Note that if $\mathrm{A} \in \mathbb{R}^{k \times n}$ has no zero entry, then $\mathrm{A}_{s}=\mathrm{A}$ for all $s \subseteq[1: n]$, and therefore $p_{\mathrm{A}}^{\star}\left(x ; z_{1}, \ldots, z_{n}\right)=p_{\mathrm{A}}(x) \prod_{i=1}^{n} z_{i}$.

Matrices having as their set of rows the set of zeros of certain cyclically symmetric matrices will play an important role in the last section of this paper. With $\mathcal{S}^{n}$ denoting symmetric $n \times n$ matrices, we call A $\in \mathcal{S}^{n}$ 
cyclically symmetric (or symmetric circulant), if it satisfies $\mathrm{A}=\mathrm{P}^{\top} \mathrm{AP}$, where $\mathrm{P}=\left(p_{i j}\right)$ is the $n \times n$ matrix satisfying $p_{i j}=1$ for $j \equiv i+1(\bmod n)$, and otherwise $p_{i j}=0$. That is, the vector $\mathbf{P v}$ is a cyclic shift of $\mathbf{v} \in \mathbb{R}^{n}$ to the top by one position. We employ the notations $\mathcal{C}^{n}:=\left\{\mathrm{A} \in \mathcal{S}^{n}: \mathrm{A}\right.$ is cyclically symmetric $\}$, and $\mathbf{C}(\mathbf{a})$ for a cyclically symmetric matrix whose first column is $\mathbf{a}$.

Note that $\mathbf{z} \in \mathbb{R}_{+}^{n}$ is a zero of a copositive form $P_{\mathrm{B}}$, where $\mathrm{B} \in \mathcal{C O} \mathcal{P}_{n, d}$ iff $\mathbf{z}$ is a global minimizer of $P_{\mathrm{B}}$ over $\Delta^{n}$ with objective value $P_{\mathrm{B}}(\mathbf{z})=0$. Optimality conditions for (strict) local minimizers of $P_{\mathrm{B}}$ can be found in [4,5] for $d=2$ and in [1] for $d>2$. In the case $d=2$, a local minimizer $\mathbf{z}$ of $P_{\mathrm{B}}(\mathbf{x})=\mathbf{x}^{\top} \mathrm{Bx}$ is said to enjoy strict complementarity, if $(\mathbf{B z})_{i}>\mathbf{z}^{\top} \mathrm{Bz}$ for any $i \notin I(\mathbf{z})$. Note that the Karush-Kuhn-Tucker conditions at $\mathbf{z}$ ensure $(\mathrm{Bz})_{i}=\mathbf{z}^{\top}$ Bz for any $i \in I(\mathbf{z})$.

TheOREM 3.7. Let the rows of $\mathrm{U} \in \mathbb{R}^{n \times n}$ be all different and coincide with the global minimizers of $P_{\mathrm{B}}$ over $\Delta^{n}$ for some $\mathrm{B} \in \mathcal{C}^{n}$, with all those minimizers conforming to strict complementarity, and with all rows of $\mathrm{U}$ being cyclic shifts of the first row $\mathbf{u}^{\top}$ of $\mathrm{U}$. Then the following holds.

a) If $n$ is prime, and the entries of $\mathrm{U}$ are rational numbers, then $\operatorname{rank} \mathrm{U}=n$.

b) If $n \geq 3$ is odd and $|I(\mathbf{u})|=n-2$, then $\operatorname{rank} \mathrm{U}=n$.

c) If $n \geq 4$ is even and $I(\mathbf{u})=[3: n]$, then $\operatorname{rank} \mathrm{U}=n-1$, but any proper subset of the set of rows of $\mathrm{U}$ has full rank. Furthermore, $\operatorname{rank}^{(2)} \mathrm{U}=n$.

d) If $n \geq 3$ and $I(\mathbf{u})=[3: n]$, then, with $\epsilon_{n}=\left\{\begin{array}{l}2, \text { for } n \text { even, } \\ 1, \text { for } n \text { odd, }\end{array}\right.$ we have

$$
p_{\mathrm{U}}^{\star}\left(x ; z_{1}, \ldots, z_{n}\right)=1+(n-2) x+x^{\epsilon_{n}}+\sum_{\substack{\phi \neq s \subseteq[1: n] \\ K_{s}(\mathrm{U}) \neq \emptyset}}\left(1+\left(\left|K_{s}(\mathrm{U})\right|-1\right) x\right) \prod_{i \in s}\left(z_{i}-1\right) .
$$

Proof. a) Not all the entries of $\mathbf{u}$ can be equal, for otherwise all rows of $U$ would coincide. Also the sum of the entries of $\mathbf{u}$ is 1 . Therefore, $\mathbf{U}$ is a circulant matrix with nonconstant rows and nonzero row sums, and [21, Proposition 23] can be employed to deduce that $\operatorname{det} \mathrm{U} \neq 0$, which implies $\operatorname{rank} \mathrm{U}=n$.

b) Let $v=\mathbf{u}^{\top} \mathrm{Bu}$ and $\overline{\mathrm{B}}=\mathrm{B}-v \boldsymbol{\eta}_{n} \boldsymbol{\eta}_{n}^{\top} \in \mathcal{C}^{n} \cap \mathcal{C O} \mathcal{P}_{n}$. Then $\mathbf{u}$ and its cyclic shifts are the zeros of $P_{\overline{\mathrm{B}}}$. Let $i, j \in[1: n]$ be those two indices not contained in $I(\mathbf{u})$. Then, there is $c>0$ such that $\mathbf{v}:=\overline{\mathrm{B}} \mathbf{u}$ satisfies $v_{i}=v_{j}=c$ and $v_{k}=0$ for $k \in I(\mathbf{u})$. Strict complementarity implies $v_{i}, v_{j}>0$, and $v_{i}=v_{j}$ is justified as follows: Define a permutation $\pi$ on $[1: n]$ by $\pi(k) \equiv i+j-k(\bmod n)$, and note that $\pi$ swaps $i$ and $j$, but leaves the set $I(\mathbf{u})$ unchanged: $\{\pi(k): k \in I(\mathbf{u})\}=I(\mathbf{u})$. Let the permutation matrix $\mathrm{R}$ satisfy $\mathrm{Re}_{k}=\mathbf{e}_{\pi(k)}$ for $k \in[1: n]$. Then $\mathrm{R}^{\top}=\mathrm{R}$, and by cyclic symmetry, we have $\mathrm{R} \overline{\mathrm{B}} \mathrm{R}=\overline{\mathrm{B}}$ which further implies $\mathbf{u}^{\top} \overline{\mathrm{B}} \mathbf{u}=\mathbf{u}^{\top} \mathrm{R} \overline{\mathrm{B}} \mathbf{R} \mathbf{u}$. Therefore $\mathbf{u}$ and $\mathrm{Ru}$ are both minimizers of $P_{\overline{\mathrm{B}}}$ over $\Delta^{n}$ with identical supports, so they must be equal. (Since we are minimizing a quadratic function, there cannot exist two different local minimizers with the same support, unless there are infinitely many such minimizers.) Thus, $(\bar{B} \mathbf{u})_{i}=(\mathrm{R} \overline{\mathrm{B} R} \mathbf{u})_{i}=(\mathrm{R} \overline{\mathrm{B}} \mathbf{u})_{i}=\mathbf{e}_{i}^{\top} \mathrm{R} \overline{\mathrm{B}} \mathbf{u}=\mathbf{e}_{\pi(i)}^{\top} \overline{\mathrm{B}} \mathbf{u}=\mathbf{e}_{j}^{\top} \overline{\mathrm{B}} \mathbf{u}=(\bar{B} \mathbf{u})_{j}$.

Let $m$ be the greatest common divisor of $n$ and $j-i$. Note that $m$ and $\ell:=n / m$ are both odd numbers. Now assume that there is $\lambda=\left[\lambda_{1}, \ldots, \lambda_{n}\right]^{\top} \in \mathbb{R}^{n} \backslash\{\mathbf{o}\}$ such that $\mathbf{o}=\sum_{k=1}^{n} \lambda_{k} \mathrm{P}^{k} \mathbf{u}$. Then, multiplying from the left by $\bar{B}$, we obtain

$$
\mathbf{o}=\sum_{k=1}^{n} \lambda_{k} \overline{\mathrm{B}} \mathrm{P}^{k} \mathbf{u}=\sum_{k=1}^{n} \lambda_{k} \mathrm{P}^{k} \overline{\mathrm{B}} \mathbf{u}=c \sum_{k=1}^{n} \lambda_{k}\left(\mathbf{e}_{i-k}+\mathbf{e}_{j-k}\right)=c\left[\begin{array}{c}
\lambda_{i-1}+\lambda_{j-1} \\
\lambda_{i-2}+\lambda_{j-2} \\
\vdots \\
\lambda_{i-n}+\lambda_{j-n}
\end{array}\right],
$$

where here and in the rest of this proof indices are computed modulo $n$ with remainders in $[1: n]$. For each 
$r \in[1: m-1]$ we are led to

$$
\lambda_{r}=-\lambda_{j-i+r}=\lambda_{2(j-i)+r}=\cdots=\lambda_{(\ell-1)(j-i)+r}=-\lambda_{r},
$$

from which we deduce $\lambda_{k m+r}=0$, for all $0 \leq k<\ell$, i.e., we have shown $\lambda_{k}=0$ for all $k \in[1: n]$. Therefore, $\operatorname{rank} \mathrm{U}=n$.

c) Proceeding as in the proof of b), we have $i=1, j=2, m=1$, and $\ell=n$ is now even. Therefore the chain of equations $\lambda_{1}=-\lambda_{2}=\lambda_{3}=\cdots=-\lambda_{n}$ has a solution depending on one parameter. We deduce, that the cokernel of $\mathrm{U}$ is spanned by the vector $\mathbf{w}:=[-1,1,-1,1, \ldots,-1,1]^{\top} \in \mathbb{R}^{n}$, so we have $\operatorname{rank} \mathrm{U}=n-1$. Since $|I(\mathbf{w})|=n$, any proper subset of the set of rows of $\mathrm{U}$ has full rank. Noting that no two rows of $\mathrm{U}$ are proportional and $\mathrm{U}$ has corank 1 , we get by Proposition 2.5 that $\operatorname{rank}^{(2)} \mathrm{U}=n$, see also [19, Lemma A.3].

d) It follows from b) and c) that $p_{\mathrm{U}}(x)=1+(n-2) x+x^{\epsilon_{n}}$. Moreover, since proper subsets of the set of rows of $\mathrm{U}$ always have full rank by b) and c), we have rank $\mathrm{U}_{s}=\left|K_{s}(\mathrm{U})\right|$, and therefore $p_{\mathrm{U}_{s}}(x)=$ $1+\left(\left|K_{s}(\mathrm{U})\right|-1\right) x$, for $K_{s}(\mathrm{U}) \neq \emptyset$.

EXAMPLE 3.8. The set of rows of the matrix

$$
\mathrm{U}:=\frac{1}{11} \mathrm{C}\left([5,3,0,0,3]^{\top}\right)=\frac{1}{11}\left[\begin{array}{ccccc}
5 & 3 & 0 & 0 & 3 \\
3 & 5 & 3 & 0 & 0 \\
0 & 3 & 5 & 3 & 0 \\
0 & 0 & 3 & 5 & 3 \\
3 & 0 & 0 & 3 & 5
\end{array}\right]
$$

is precisely the set of global minimizers of $\mathrm{B}:=\mathrm{C}\left([3,0,2,2,0]^{\top}\right) \in \mathcal{C}^{5}$ (and the set of zeros of $\mathrm{B}-\frac{15}{11} \boldsymbol{\eta}_{5} \boldsymbol{\eta}_{5}^{\top} \in \mathcal{C}^{5} \cap \mathcal{C O P}_{5}$ ), and those conform to strict complementarity. Another such pair (of order $7)$ is $\mathrm{B}=\mathrm{C}\left([4,0,1,2,2,1,0]^{\top}\right), \mathrm{U}=\frac{1}{66} \mathrm{C}\left([20,16,7,0,0,7,16]^{\top}\right)$.

Note that matrices $\mathrm{U}$ as in Theorem $3.7 \mathrm{~d}$ ) exist for any $n \geq 3$, see [11, Theorem 5] for a construction using $\mathrm{B}:=\mathrm{C}\left([2 \cos \theta,-1,0, \ldots, 0,-1]^{\top}\right) \in \mathcal{C}^{n}$ with $\frac{2 \pi}{n}<\theta<\frac{2 \pi}{n-1}$.

In Section 7 , for $\mathrm{U} \in \mathbb{R}^{n \times n}$ as in Theorem 3.7, we will need to evaluate $p_{\mathrm{U}}^{\star}\left(x ; z_{1}, \ldots, z_{n}\right)$ at polynomials $z_{i}=p_{\mathrm{V}_{i}}(x)$. The following corollary will be of help in that regard.

Corollary 3.9. For fixed $n \geq 5$ let $0<m<n$ and let $\mathrm{U}$ satisfy the conditions of Theorem 3.7 $d$ ), and let $a, b$ be polynomials in $x$. Then, up to terms of order 3 ,

$$
\begin{aligned}
p_{\mathrm{U}}^{\star}(x ; \underbrace{1+a x, \ldots, 1+a x}_{m \text { times }}, \underbrace{1+b x, \ldots, 1+b x}_{n-m \text { times }})= & 1+(n-2) x+x^{\epsilon_{n}}+(1+(n-3) x)(m a+(n-m) b) x \\
& +\frac{1}{2}(m a+(n-m) b)^{2} x^{2}-\frac{1}{2}\left(m a^{2}+(n-m) b^{2}\right) x^{2}+\mathcal{O}\left(x^{3}\right) .
\end{aligned}
$$

Proof. Note that $n \geq 5$ ensures that $K_{s}(\mathrm{U}) \neq \emptyset$ for $s \subseteq[1: n]$ satisfying $|s| \leq 2$. More precisely, if $|s|=1$ then $\left|K_{s}(\mathrm{U})\right|=n-2$. If $s=\{1,2\}$ or any of its cyclic shifts, then $\left|K_{s}(\mathrm{U})\right|=n-3$, and in all other 
cases of $|s|=2$, we have $\left|K_{s}(\mathrm{U})\right|=n-4$. This results in

$$
\begin{aligned}
p_{\mathrm{U}}^{\star}(x ; 1+a x, \ldots, 1+a x, 1+b x, \ldots, 1+b x)= & p_{\mathrm{U}}(x)+p_{\mathrm{U}_{\{1\}}}(x)(m a+(n-m) b) x \\
& +p_{\mathrm{U}_{\{1,2\}}}(x)\left((m-1) a^{2}+(n-m-1) b^{2}+2 a b\right) x^{2} \\
& +p_{\mathrm{U}_{\{1,3\}}}(x)\left(\left(\begin{array}{c}
m-1 \\
2
\end{array}\right) a^{2}+\left(\begin{array}{c}
n-m-1 \\
2
\end{array}\right) b^{2}+(m(n-m)-2) a b\right) x^{2} \\
& +\mathcal{O}\left(x^{3}\right) \\
= & +(n-2) x+x^{\epsilon_{n}}+(1+(n-3) x)(m a+(n-m) b) x \\
& +\left(\left(\begin{array}{c}
m \\
2
\end{array}\right) a^{2}+\left(\begin{array}{c}
n-m \\
2
\end{array}\right) b^{2}+m(n-m) a b\right) x^{2}+\mathcal{O}\left(x^{3}\right),
\end{aligned}
$$

using $p_{\mathrm{U}_{\{1,2\}}}(x)=1+(n-4) x=1+\mathcal{O}(x), p_{\mathrm{U}_{\{1,3\}}}(x)=1+(n-5) x=1+\mathcal{O}(x)$, and a final rearrangement completes the proof.

4. A certain product and its relation to generating polynomials. We will first recall the operation $\otimes$, that has been introduced in [10] and related to the Kathri-Rao product (see [20]), and that turns an important set of matrices into a semigroup, with generating polynomials giving rise to a homomorphism.

For matrices $\mathrm{U} \in \mathbb{R}^{k \times n}$ and $\mathrm{V} \in \mathbb{R}^{\ell \times m}$ we construct the following $k \ell \times(n+m)$ matrix, whose rows are all the combinations $\left[\mathbf{u}^{\top}, \mathbf{v}^{\top}\right]$ of rows $\mathbf{u}^{\top}$ of $\mathrm{U}$ and rows $\mathbf{v}^{\top}$ of $\mathrm{V}$, denoted as $\mathrm{U} \otimes \mathrm{V}=\left[\mathrm{U} \otimes \boldsymbol{\eta}_{\ell} \mid \boldsymbol{\eta}_{k} \otimes \mathrm{V}\right]$ Furthermore, in multiple products, we start multiplying from the left, $\mathrm{U} \oplus \mathrm{V} \oplus \mathrm{W}:=(\mathrm{U} \oplus \mathrm{V}) \oplus \mathrm{W}$ etc. and note that changing the order in which multiplications are carried out would result in a permutation of the rows of the resulting product.

Example 4.1. If $A=\left[\begin{array}{ll}1 & 0 \\ 0 & 3\end{array}\right]$ and $B=\left[\begin{array}{lll}1 & 1 & 1 \\ 2 & 1 & 2\end{array}\right]$, then $A \oplus B=\left[\begin{array}{lllll}1 & 0 & 1 & 1 & 1 \\ 1 & 0 & 2 & 1 & 2 \\ 0 & 3 & 1 & 1 & 1 \\ 0 & 3 & 2 & 1 & 2\end{array}\right]$.

THEOREM 4.2. Let $\mathrm{A} \in \mathbb{R}^{N_{\mathrm{A}} \times n_{\mathrm{A}}}, \mathrm{B} \in \mathbb{R}^{N_{\mathrm{B}} \times n_{\mathrm{B}}}$ share the following properties:

(i) the rows are pairwise linearly independent,

(ii) the all ones vector is contained in the column space.

Then $\mathrm{A} \otimes \mathrm{B}$ satisfies (i) and (ii) as well, and $p_{\mathrm{A} \otimes \mathrm{B}}(x)=p_{\mathrm{A}}(x) p_{\mathrm{B}}(x)$.

Proof. We may assume that both $\mathrm{A}$ and $\mathrm{B}$ are row-adjusted, with $\ell+1$, resp., $m+1$, blocks, individual blocks $\mathrm{A}_{i}$, resp., $\mathrm{B}_{i}$, having $a_{i}$, resp., $b_{i}$ rows. Because of (ii) there are $\boldsymbol{\alpha} \in \mathbb{R}^{n_{\mathrm{A}}}$ and $\boldsymbol{\beta} \in \mathbb{R}^{n_{\mathrm{B}}}$ such that $\mathrm{A} \boldsymbol{\alpha}=\boldsymbol{\eta}_{N_{\mathrm{A}}}, \mathrm{B} \boldsymbol{\beta}=\boldsymbol{\eta}_{N_{\mathrm{B}}}$. We let $\mathrm{C}:=\mathrm{A} \otimes \mathrm{B}$ and observe that $\mathrm{C}$ satisfies (i) and (ii). Because of (i), up to a permutation of the rows, $C$ is equal to some row-adjusted matrix $\left[\mathrm{C}_{0}^{\top}|\cdots| \mathrm{C}_{\ell+m}^{\top}\right]^{\top}$, with $c_{k}$ the number of rows of $C_{k}$. We claim that we may use

$$
C_{0}=A_{0} \otimes B_{0}, \quad C_{1}=\left[\begin{array}{l}
A_{0} \otimes B_{1} \\
A_{1} \circledast B_{0}
\end{array}\right], \quad C_{2}=\left[\begin{array}{l}
A_{0} \oplus B_{2} \\
A_{1} \circledast B_{1} \\
A_{2} \oplus B_{0}
\end{array}\right], \quad \text { etc. }
$$

with $\mathrm{C}_{k}$ a stack of matrices $\left(\mathrm{A}_{i} \otimes \mathrm{B}_{k-i}\right)_{i \in[\max (0, k-m): \min (k, \ell)]}$ for $k \in[0: \ell+m]$. It is easy to see that the row sets of $\left(C_{k}\right)_{k \in[0: \ell+m]}$ indeed constitute a partition of the row set of $C$. We note for further use that for given matrices $\mathrm{U}, \mathrm{V}$ any of the matrices $\left(\mathrm{U}^{\langle i\rangle} \otimes \mathrm{V}^{\langle k-i\rangle}\right)_{i \in[0: k]}$ is a submatrix of $(\mathrm{U} \otimes \mathrm{V})^{\langle k\rangle}$, with all those matrices having the same number of rows, and that $\operatorname{rank}(\mathrm{U} \otimes \mathrm{V})=\operatorname{rank} \mathrm{U} \operatorname{rank} \mathrm{V}$ holds. For the proof of $\mathrm{C}_{[0: \ell+m]}$ being row-adjusted, we proceed in two steps. 
Step 1: For $k \in[0, \ell+m]$, we have $\operatorname{rank}^{(k)} \mathrm{C}_{[0: k]}=\sum_{i=0}^{k} c_{i}$ :

In order to show that $C_{[0: k]}^{\langle k\rangle}$ has full row rank, we let $\mathcal{N}:=[\max (0, k-m): \min (k, \ell)]$ and assume that there are vectors $\left(\mathbf{u}_{i}\right)_{i \in \mathcal{N}}$ of fitting dimension such that

$$
\sum_{i \in \mathcal{N}} \mathbf{u}_{i}^{\top}\left(\mathrm{A}_{[0: i]} \otimes \mathrm{B}_{k-i}\right)^{\langle k\rangle}=\mathbf{o}^{\top} .
$$

Assuming that for some $j \in \mathcal{N}$ we have already shown that $\mathbf{u}_{i}=\mathbf{o}$ for $i<j$, we are led to $\sum_{i \in \mathcal{N}_{j}} \mathbf{u}_{i}^{\top}\left(\mathrm{A}_{[0: i]} \otimes \mathrm{B}_{k-i}\right)^{\langle k\rangle}=\mathbf{o}^{\top}$, where $\mathcal{N}_{j}:=\mathcal{N} \cap[j: \ell+m]$. It follows that $\sum_{i \in \mathcal{N}_{j}} \mathbf{u}_{i}^{\top}\left(\mathrm{A}_{[0: i]}^{\langle j\rangle} \otimes \mathrm{B}_{k-i}^{\langle k-j\rangle}\right)=\mathbf{o}^{\top}$ holds. Since $A$ is row-adjusted, for any $i \in \mathcal{N}_{j}$ there is a matrix $Q_{i j}$ such that $A_{[0: i]}^{\langle j\rangle}=Q_{i j} A_{[0: j]}^{\langle j\rangle}$ holds. Then, with identity matrix I of fitting size,

$$
\mathrm{A}_{[0: i]}^{\langle j\rangle} \otimes \mathrm{B}_{k-i}^{\langle k-j\rangle}=\mathrm{Q}_{i j} \mathrm{~A}_{[0: j]}^{\langle j\rangle} \otimes I \mathrm{~B}_{k-i}^{\langle k-j\rangle}=\left(\mathrm{Q}_{i j} \otimes \mathrm{I}\right)\left(\mathrm{A}_{[0: j]}^{\langle j\rangle} \otimes \mathrm{B}_{k-i}^{\langle k-j\rangle}\right) .
$$

So, denoting $\mathbf{v}_{i}^{\top}:=\mathbf{u}_{i}^{\top}\left(Q_{i j} \otimes \mathrm{I}\right)$ for $i \in \mathcal{N}_{j}$, and $\mu:=\max (0, k-\ell)$, we arrive at

$$
\mathbf{u}_{j}^{\top}\left(\mathrm{A}_{[0: j]}^{\langle j\rangle} \otimes \mathrm{B}_{k-j}^{\langle k-j\rangle}\right)+\sum_{i \in \mathcal{N}_{j}, i>j} \mathbf{v}_{i}^{\top}\left(\mathrm{A}_{[0: j]}^{\langle j\rangle} \otimes \mathrm{B}_{k-i}^{\langle k-j\rangle}\right)=\mathbf{w}^{\top}\left(\mathrm{A}_{[0: j]}^{\langle j\rangle} \otimes \mathrm{B}_{[\mu: k-j]}^{\langle k-j\rangle}\right)=\mathbf{o}^{\top},
$$

where the components of $\mathbf{w}^{\top}$ are permuted components of $\left[\mathbf{u}_{j}^{\top}, \mathbf{v}_{j+1}^{\top}, \ldots, \mathbf{v}_{\min (k, \ell)}^{\top}\right]$. Since $\mathrm{A}_{[0: j]}^{\langle j\rangle} \otimes \mathrm{B}_{[\mu: k-j]}^{\langle k-j\rangle}$ has full row rank by our assumptions, we have $\mathbf{w}=\mathbf{o}$ and therefore $\mathbf{u}_{j}=\mathbf{o}$. By induction, we conclude that $\mathbf{u}_{i}=\mathbf{o}$ for every $i \in \mathcal{N}$. Therefore $\mathrm{C}_{[0: k]}^{\langle k\rangle}$, which is, up to a permutation of the rows, a stack of matrices $\left(\left(\mathrm{A}_{[0: i]} \oplus \mathrm{B}_{k-i}\right)^{\langle k\rangle}\right)_{i \in \mathcal{N}}$, has full row rank.

Step 2: For $k \in[0, \ell+m]$, we have $\operatorname{rank}^{(k)} \mathrm{C}=\operatorname{rank}^{(k)} \mathrm{C}_{[0: k]}$ :

The left hand side cannot be smaller then the right hand side, since $\mathrm{C}_{[0: k]}$ is a submatrix of $\mathrm{C}$. It cannot be larger either since rowsp $C^{\langle k\rangle} \subseteq$ rowsp $C_{[0, k]}^{\langle k\rangle}$. We will prove the latter property of row spaces by showing

$$
K>k \Longrightarrow \operatorname{rowsp} \mathrm{C}_{[0, K]}^{\langle k\rangle} \subseteq \operatorname{rowsp} \mathrm{C}_{[0, K-1]}^{\langle k\rangle} .
$$

We will employ the following two lemmas.

Lemma 4.3. Let $k \in \mathbb{N}$, $\mathbf{a}^{\top}$ a row of $\mathrm{A}, \mathbf{b}^{\top}$ a convex combination of rows of $\mathrm{B}$, and $\boldsymbol{\lambda} \in \mathbb{R}^{N_{\mathrm{B}}}$ such that $\left(\mathbf{b}^{\top}\right)^{\langle k\rangle}=\boldsymbol{\lambda}^{\top} \mathrm{B}^{\langle k\rangle}$. Then $\left(\mathbf{a}^{\top} \otimes \mathbf{b}^{\top}\right)^{\langle k\rangle}=\boldsymbol{\lambda}^{\top}\left(\mathbf{a}^{\top} \otimes \mathrm{B}\right)^{\langle k\rangle}$.

Proof. Note that $\mathbf{b}^{\top}=\boldsymbol{\mu}^{\top} \mathrm{B}$ with $\boldsymbol{\mu} \in \Delta^{N_{B}}$ implies $\mathbf{b}^{\top} \boldsymbol{\beta}=\boldsymbol{\mu}^{\top} \mathrm{B} \boldsymbol{\beta}=\boldsymbol{\mu}^{\top} \boldsymbol{\eta}_{N_{B}}=1$. Next, observe that $\left(\mathbf{a}^{\top} \otimes \mathbf{b}^{\top}\right)^{\langle k\rangle}$ can be decomposed into $2^{k}$ submatrices, where to any $\bar{k} \in[0: k]$ and any subset of $[1: k]$ of size $\bar{k}$ there corresponds a submatrix built from $\bar{k}$ factors $\mathbf{b}^{\top}$ and $k-\bar{k}$ factors $\mathbf{a}^{\top}$ in an order determined by that subset. Up to a permutation of the columns, that submatrix equals $\left(\mathbf{a}^{\top}\right)^{\langle k-\bar{k}\rangle} \otimes\left(\mathbf{b}^{\top}\right)^{\langle\bar{k}\rangle}$. So, we are left with showing that $\left(\mathbf{a}^{\top}\right)^{\langle k-\bar{k}\rangle} \otimes\left(\mathbf{b}^{\top}\right)^{\langle\bar{k}\rangle}=\boldsymbol{\lambda}^{\top}\left(\left(\mathbf{a}^{\top}\right)^{\langle k-\bar{k}\rangle} \otimes \mathrm{B}^{\langle\bar{k}\rangle}\right)$, which would follow from $\left(\mathbf{b}^{\top}\right)^{\langle\bar{k}\rangle}=\boldsymbol{\lambda}^{\top} \mathbf{B}^{\langle\bar{k}\rangle}$ holding for $\bar{k} \in[0: k]$. Now the latter equality holds for $\bar{k}=k$ by assumption. Assuming, it has been shown for $\bar{k}+1 \in[1: k]$, we prove it for $\bar{k}$ via

$$
\begin{aligned}
\left(\mathbf{b}^{\top}\right)^{\langle\bar{k}\rangle} & =\left(\mathbf{b}^{\top} \boldsymbol{\beta}\right)\left(\mathbf{b}^{\top}\right)^{\langle\bar{k}\rangle}=\left(\left(\mathbf{b}^{\top}\right)^{\langle\bar{k}\rangle} \otimes \mathbf{b}^{\top}\right)\left(\mathrm{I}_{n_{\mathrm{B}}^{\bar{B}}} \otimes \boldsymbol{\beta}\right)=\left(\mathbf{b}^{\top}\right)^{\langle\bar{k}+1\rangle}\left(\mathrm{I}_{n_{\mathrm{B}} \overline{\bar{k}}} \otimes \boldsymbol{\beta}\right)=\boldsymbol{\lambda}^{\top} \mathrm{B}^{\langle\bar{k}+1\rangle}\left(\mathrm{I}_{n_{\mathrm{B}} \overline{\bar{k}}} \otimes \boldsymbol{\beta}\right) \\
& =\sum_{i=1}^{N_{B}} \lambda_{i}\left(\left(\mathbf{e}_{i}^{\top} \mathrm{B}\right)^{\langle\bar{k}\rangle} \otimes\left(\mathbf{e}_{i}^{\top} \mathrm{B}\right)\right)\left(\mathrm{I}_{n_{\mathrm{B}}^{\bar{k}}} \otimes \boldsymbol{\beta}\right)=\sum_{i=1}^{N_{B}} \lambda_{i}\left(\mathbf{e}_{i}^{\top} \mathrm{B}\right)^{\langle\bar{k}\rangle}\left(\mathbf{e}_{i}^{\top} \mathrm{B} \boldsymbol{\beta}\right)=\boldsymbol{\lambda}^{\top} \mathrm{B}^{\langle\bar{k}\rangle} .
\end{aligned}
$$

This completes a proof by induction. 
Electronic Journal of Linear Algebra, ISSN 1081-3810

A publication of the International Linear Algebra Society

Volume 36, pp. 519-541, August 2020.

LEMmA 4.4. Let $i, j, k \geq 0, i+j \geq k-1, \mathbf{a}^{\top}$ a row of $\mathrm{A}$ and $\mathbf{b}^{\top}$ a row of $\mathrm{B}$, and $\boldsymbol{\lambda}, \boldsymbol{\mu}$ of fitting dimension satisfying $\left(\mathbf{a}^{\top}\right)^{\langle i\rangle}=\boldsymbol{\lambda}^{\top} \mathrm{A}_{[0: i]}^{\langle i\rangle},\left(\mathbf{b}^{\top}\right)^{\langle j\rangle}=\boldsymbol{\mu}^{\top} \mathrm{B}_{[0: j]}^{\langle j\rangle}$. Then

$$
\left(\mathbf{a}^{\top} \otimes \mathbf{b}^{\top}\right)^{\langle k\rangle}=\boldsymbol{\lambda}^{\top}\left(\mathrm{A}_{[0: i]} \circledast \mathbf{b}^{\top}\right)^{\langle k\rangle}+\boldsymbol{\mu}^{\top}\left(\mathbf{a}^{\top} \otimes \mathrm{B}_{[0: j]}\right)^{\langle k\rangle}-(\boldsymbol{\lambda} \otimes \boldsymbol{\mu})^{\top}\left(\mathrm{A}_{[0: i]} \circledast \mathrm{B}_{[0: j]}\right)^{\langle k\rangle} .
$$

Proof. We use an analogous decomposition into $2^{k}$ submatrices as in the proof of Lemma 4.3, which necessitates investigation of the right hand side term

$$
\boldsymbol{\lambda}^{\top}\left(\mathrm{A}_{[0: i]}^{\langle k-\bar{k}\rangle} \otimes\left(\mathbf{b}^{\top}\right)^{\langle\bar{k}\rangle}\right)+\boldsymbol{\mu}^{\top}\left(\left(\mathbf{a}^{\top}\right)^{\langle k-\bar{k}\rangle} \otimes \mathrm{B}_{[0: j]}^{\langle\bar{k}\rangle}\right)-\boldsymbol{\lambda}^{\top} \mathrm{A}_{[0: i]}^{\langle k-\bar{k}\rangle} \otimes \boldsymbol{\mu}^{\top} \mathrm{B}_{[0: j]}^{\langle\bar{k}\rangle} .
$$

Note that $\bar{k} \in[0: k]$ implies either $k-\bar{k} \leq i$ or $\bar{k} \leq j$. As in the proof of Lemma 4.3 we have $\lambda^{\top} \mathrm{A}_{[0: i]}^{\langle k-\bar{k}\rangle}=$ $\left(\mathbf{a}^{\top}\right)^{\langle k-\bar{k}\rangle}$ for $k-\bar{k} \in[0: i]$, and $\boldsymbol{\mu}^{\top} \mathrm{B}_{[0: j]}^{\langle\bar{k}\rangle}=\left(\mathbf{b}^{\top}\right)^{\langle\bar{k}\rangle}$ for $\bar{k} \in[0: j]$. Therefore, second and third term in (4.3) cancel out if $k-\bar{k} \leq i$, and first and third term in (4.3) cancel out if $\bar{k} \leq j$, leaving $\left(\mathbf{a}^{\top}\right)^{\langle k-\bar{k}\rangle} \otimes\left(\mathbf{b}^{\top}\right)^{\langle\bar{k}\rangle}$ in both cases, which is the corresponding left hand side term.

Continuing with the proof of (4.2), we assume $K>k$ and let $\mathbf{c}^{\top}$ be a row of $\mathrm{C}_{[0: K]}$. Then there are $i_{\circ}, j_{\circ} \geq 0$ satisfying $i_{\circ}+j_{\circ}=K$, such that $\mathbf{c}^{\top}=\mathbf{a}^{\top} \otimes \mathbf{b}^{\top}$, where $\mathbf{a}^{\top}$ is a row of $\mathrm{A}_{\left[0: i_{\circ}\right]}$ and $\mathbf{b}^{\top}$ is a row of $\mathrm{B}_{\left[0: j_{\circ}\right]}$. When $i_{\circ}=0$, we use $\left(\mathbf{b}^{\top}\right)^{\langle k\rangle}=\boldsymbol{\lambda}^{\top} \mathrm{B}_{[0: k]}^{\langle k\rangle}$ with suitable $\boldsymbol{\lambda}$, and Lemma 4.3 to deduce

$$
\left(\mathbf{c}^{\top}\right)^{\langle k\rangle} \in \operatorname{rowsp}\left(\mathbf{a}^{\top} \circledast \mathrm{B}_{[0, k]}\right)^{\langle k\rangle} \subseteq \operatorname{rowsp} C_{[0, k]}^{\langle k\rangle} \subseteq \operatorname{rowsp} C_{[0, K-1]}^{\langle k\rangle} .
$$

We also find $\left(\mathbf{c}^{\top}\right)^{\langle k\rangle} \in \operatorname{rowsp} C_{[0, K-1]}^{\langle k\rangle}$ when $j_{\circ}=0$. Assuming now $\min \left(i_{\circ}, j_{\circ}\right)>0$, we let $i:=i_{\circ}-1 \geq 0$ and $j:=j_{\circ}-1 \geq 0$, which satisfy $i+j \geq k-1$. Since A, B are row-adjusted, all the hypotheses of Lemma 4.4 are fulfilled, and we conclude that $\left(\mathbf{c}^{\top}\right)^{\langle k\rangle} \in \operatorname{rowsp}\left[\begin{array}{l}\mathrm{A}_{\left[0: i_{\circ}-1\right]} \otimes \mathrm{B}_{\left[0: j_{\circ}\right]} \\ \mathrm{A}_{\left[0: i_{\circ}\right]} \circledast \mathrm{B}_{\left[0: j_{\circ}-1\right]}\end{array}\right]^{\langle k\rangle} \subseteq \operatorname{rowsp} \mathrm{C}_{[0, K-1]}^{\langle k\rangle}$. This completes Step 2.

Since $\mathrm{A}_{[0: k]} \otimes \mathrm{B}$ is a submatrix of $\mathrm{C}$ and $\mathrm{C}_{[0: k]}$ is a submatrix of $\mathrm{A}_{[0: k]} \circledast \mathrm{B}$, the assertion made in Step 2 implies

$$
\operatorname{rank}^{(k)} \mathrm{C}=\operatorname{rank}^{(k)}\left(\mathrm{A}_{[0: k]} \circledast \mathrm{B}\right)
$$

noted for later use. By our construction, $p_{\mathrm{A} \oplus \mathrm{B}}(x)=p_{\mathrm{C}}(x)=\sum_{i=0}^{\ell+m} c_{i} x^{i}$, where the coefficient $\left[x^{k}\right] p_{\mathrm{C}}(x)=$ $c_{k}=\operatorname{rank}^{(k)} \mathrm{C}_{k}=\sum_{i=\max (0, k-m)}^{\min (k, \ell)} a_{i} b_{k-i}=\left[x^{k}\right] p_{\mathrm{A}}(x) p_{\mathrm{B}}(x)$, which shows $p_{\mathrm{A} \circledast \mathrm{B}}(x)=p_{\mathrm{A}}(x) p_{\mathrm{B}}(x)$.

REMARK 4.5. Equality of constant and linear terms, and an inequality for the coefficients of $x^{2}$, of $p_{\mathrm{A} \oplus \mathrm{B}}(x)$ and $p_{\mathrm{A}}(x) p_{\mathrm{B}}(x)$ was shown in $[10$, Lemma $15 \mathrm{ab}]$. For $p_{\mathrm{A} \circledast \mathrm{B}}(x)=p_{\mathrm{A}}(x) p_{\mathrm{B}}(x)$ to hold, property (ii) is indispensable; see the following example: Let $A:=I_{1}$ and $B:=\left[\begin{array}{ll}1 & 1 \\ 1 & 3 \\ 2 & 1 \\ 2 & 3\end{array}\right]$, with $\boldsymbol{\eta}_{4} \notin$ colsp $B$, and $\mathrm{C}:=\mathrm{A} \otimes \mathrm{B}=\left[\begin{array}{lll}1 & 1 & 1 \\ 1 & 1 & 3 \\ 1 & 2 & 1 \\ 1 & 2 & 3\end{array}\right]$. Then $p_{\mathrm{C}}(x)=(1+x)^{2} \neq 1+x+x^{2}+x^{3}=p_{\mathrm{A}}(x) p_{\mathrm{B}}(x)$. Property (i) is however not required: For $A, B$ not both conforming to (i), let $\bar{A}, \bar{B}$ be maximal submatrices of $A$, B composed of pairwise linearly independent rows. Then $\bar{A} \oplus \bar{B}$ is a maximal submatrix of $A \circledast B$ composed of pairwise linearly independent rows. So, if both $\mathrm{A}, \mathrm{B}$ (and hence, $\overline{\mathrm{A}}, \overline{\mathrm{B}}$ ) conform to (ii), we have $p_{\mathrm{A} \circledast \mathrm{B}}(x)=p_{\overline{\mathrm{A}} \circledast \overline{\mathrm{B}}}(x)=$ $p_{\overline{\mathrm{A}}}(x) p_{\overline{\mathrm{B}}}(x)=p_{\mathrm{A}}(x) p_{\mathrm{B}}(x)$. 
Here is a slight generalization of Theorem 4.2 .

TheOrem 4.6. Let A, B satisfy property (i) of Theorem 4.2, and let $\mathbf{u} \in \mathbb{R}^{n_{\mathrm{A}}}, \mathbf{v} \in \mathbb{R}^{n_{\mathrm{B}}}$ be such that $\mathrm{Au}$ and $\mathrm{Bv}$ have no zero components. Let $\mathrm{C}:=[\mathrm{A} \otimes \mathbf{B v} \mid \mathrm{Au} \otimes \mathrm{B}]$. Then $p_{\mathrm{C}}(x)=p_{\mathrm{A}}(x) p_{\mathrm{B}}(x)$.

Proof. By our assumptions there are nonsingular diagonal matrices $D_{A}, D_{B}$ such that $D_{A} A u=\eta_{N_{A}}$ and $\mathrm{D}_{\mathrm{B}} \mathrm{Bv}=\boldsymbol{\eta}_{N_{\mathrm{B}}}$. We use those to define $\overline{\mathrm{A}}:=\mathrm{D}_{\mathrm{A}} \mathrm{A}, \overline{\mathrm{B}}:=\mathrm{D}_{\mathrm{B}} \mathrm{B}$, and $\overline{\mathrm{C}}:=\left(\mathrm{D}_{\mathrm{A}} \otimes \mathrm{D}_{\mathrm{B}}\right) \mathrm{C}=\left[\overline{\mathrm{A}} \otimes \boldsymbol{\eta}_{N_{\mathrm{B}}} \mid \boldsymbol{\eta}_{N_{\mathrm{A}}} \otimes \overline{\mathrm{B}}\right]=\overline{\mathrm{A}} \otimes \overline{\mathrm{B}}$. Then, by Proposition $2.4 \mathrm{~d}$ ) and Theorem 4.2, $p_{\mathrm{C}}(x)=p_{\overline{\mathrm{C}}}(x)=p_{\overline{\mathrm{A}}}(x) p_{\overline{\mathrm{B}}}(x)=p_{\mathrm{A}}(x) p_{\mathrm{B}}(x)$, noting that (ii) of Theorem 4.2 is satisfied for $\overline{\mathrm{A}}, \overline{\mathrm{B}}$, because of $\overline{\mathrm{A}} \mathbf{u}=\boldsymbol{\eta}_{N_{\mathrm{A}}}, \overline{\mathrm{B}} \mathbf{v}=\boldsymbol{\eta}_{N_{\mathrm{B}}}$.

Remark 4.7. Assuming row-adjustedness of A, B in Theorem 4.6, a row-adjusted representation of $C$ can be constructed along the lines of the proof of Theorem 4.2, with $\left[\mathrm{A}_{i} \otimes \mathrm{B}_{k-i} \mathbf{v} \mid \mathrm{A}_{i} \mathbf{u} \otimes \mathrm{B}_{k-i}\right]$ replacing $\mathrm{A}_{i} \otimes \mathrm{B}_{k-i}$. Equation (4.4) then implies

$$
\operatorname{rank}^{(k)} \mathrm{C}=\operatorname{rank}^{(k)} \overline{\mathrm{C}}=\operatorname{rank}^{(k)}\left(\overline{\mathrm{A}}_{[0: k]} \otimes \overline{\mathrm{B}}\right)=\operatorname{rank}^{(k)}\left[\mathrm{A}_{[0: k]} \otimes \mathrm{Bv} \mid \mathrm{A}_{[0: k]} \mathbf{u} \otimes \mathrm{B}\right] .
$$

Furthermore, if A, B of Theorem 4.6 additionally satisfy property $($ ii $)$ of Theorem 4.2 , then we have $\rho(\mathrm{C})=$ $\rho(\mathrm{A} \otimes \mathrm{B})$.

5. Constructing complicated zero sets from simpler ones. Here we will introduce for any matrix $\mathrm{U}$ a further operation, with as many operands (again matrices) as the number of columns of $U$. If all involved matrices are composed of rows from some zero sets of copositive forms of the same degree, then the result of the operation will also be such a matrix, and its generating polynomial can be expressed in terms of the generating polynomials of the involved matrices, which will be the content of Theorem 5.3.

For a $k \times n$ matrix $\mathrm{A}$ and $\alpha \in \mathbb{R}$, we define

$$
\alpha \star \mathrm{A}:= \begin{cases}\alpha \mathrm{A}, & \alpha \neq 0, \\ {[0, \ldots, 0] \in \mathbb{R}^{1 \times n},} & \alpha=0 .\end{cases}
$$

Next, let $\mathrm{U}$ be a $k \times n$ matrix and let for $i \in[1: n]$ the matrices $\mathrm{V}_{i}$ be $k_{i} \times m_{i}$ matrices. Define

$$
\circledast \mathrm{u}\left(\mathrm{V}_{1}, \ldots, \mathrm{V}_{n}\right):=\left[\begin{array}{c}
\left(u_{11} \star \mathrm{V}_{1}\right) \oplus \cdots \oplus\left(u_{1 n} \star \mathrm{V}_{n}\right) \\
\vdots \\
\left(u_{k 1} \star \mathrm{V}_{1}\right) \oplus \cdots \oplus\left(u_{k n} \star \mathrm{V}_{n}\right)
\end{array}\right],
$$

and observe that this matrix has $\sum_{i=1}^{n} m_{i}$ columns. Its number of rows will turn out to be $p_{\mathrm{U}}^{\star}\left(1, k_{1}, \ldots, k_{n}\right)$, in case that all matrices $\mathrm{U}, \mathrm{V}_{1}, \ldots, \mathrm{V}_{n}$ are row-stochastic and have no repeated rows.

A matrix $\mathrm{A} \in[0,1]^{k \times n}$ is called row-stochastic, if $\mathrm{A} \boldsymbol{\eta}_{n}=\boldsymbol{\eta}_{k}$. Denote by $\mathcal{R}$ the set of all row-stochastic matrices of any dimension with no repeated rows. So, matrices in $\mathcal{R}$ have properties (i) and (ii) of Theorem 4.2 . Furthermore, define for each $d \geq 2$ the following set of matrices:

$$
\mathcal{R}^{d}:=\{\mathrm{U} \in \mathcal{R}: \text { the rows of } \mathrm{U} \text { are all the zeros of some copositive form of degree } d\}
$$

REMARK 5.1. Note that $\mathrm{U} \in \mathcal{R}^{d} \Longrightarrow \mathrm{U} \in \mathcal{R}^{d^{\prime}}$ for all $d^{\prime} \geq d$ : The set of zeros of a copositive form $f(\mathbf{x})$ of degree $d$ defined for $\mathbf{x} \in \mathbb{R}^{n}$ coincides with the set of zeros of $\left(\boldsymbol{\eta}_{n}^{\top} \mathbf{x}\right)^{d^{\prime}-d} f(\mathbf{x})$, which is a copositive form of degree $d^{\prime}$.

Lemma 5.2. Let $\mathrm{U} \in \mathbb{R}^{j \times n}, \mathrm{~V} \in \mathbb{R}^{\ell \times m}$, with $\mathrm{U}, \mathrm{V} \in \mathcal{R}$. Let $\mathrm{W}:=\mathbb{U}_{\mathrm{U}}\left(\mathrm{V}, \mathrm{I}_{1}, \ldots, \mathrm{I}_{1}\right)$. Then $p_{\mathrm{W}}(x)=p_{\mathrm{U}}(x)+$ $p_{\mathrm{U}_{\{1\}}}(x)\left(p_{\vee}(x)-1\right)=p_{\mathrm{U}}^{\star}\left(x ; p_{\vee}(x), 1, \ldots, 1\right)$ holds. 
Electronic Journal of Linear Algebra, ISSN 1081-3810

More generally, with $\mathrm{V}$ at position $k$, i.e., for $\mathrm{W}_{k}:=\circledast \mathrm{U}(\underbrace{\mathrm{I}_{1}, \ldots, \mathrm{I}_{1}}_{k-1}, \mathrm{~V}, \underbrace{\mathrm{I}_{1}, \ldots, \mathrm{I}_{1}}_{n-k})$, we have $p_{\mathrm{W}_{k}}(x)=$ $p_{\mathrm{U}}(x)+p_{\mathrm{U}_{\{k\}}}(x)\left(p_{\mathrm{V}}(x)-1\right)=p_{\mathrm{U}}^{\star}(x ; \underbrace{1, \ldots, 1}_{k-1}, p_{\mathrm{V}}(x), \underbrace{1, \ldots, 1}_{n-k})$.

Proof. The equality $p_{\mathrm{U}}(x)+p_{\mathrm{U}_{\{1\}}}(x)\left(p_{\vee}(x)-1\right)=p_{\mathrm{U}}^{\star}\left(x ; p_{\vee}(x), 1, \ldots, 1\right)$ follows from the definition of $p_{\mathrm{U}}^{\star}$. To prove $p_{\mathrm{W}}(x)=p_{\mathrm{U}}(x)-p_{\mathrm{U}_{\{1\}}}(x)+p_{\mathrm{U}_{\{1\}}}(x) p_{\mathrm{V}}(x)$, we consider a special case first.

If $K_{\{1\}}(\mathrm{U})=\{\}$, i.e., $p_{\mathrm{U}_{\{1\}}}$ is the zero polynomial, then $\mathrm{W}=[\mathrm{O} \mid \mathrm{U}]$, with $\mathrm{O}$ the $j \times m-1$ zero matrix, if $m>1$, and $\mathrm{W}=\mathrm{U}$, if $m=1$, resulting in $p_{\mathrm{W}}(x)=p_{\mathrm{U}}(x)$ via Proposition $\left.2.4 \mathrm{~b}\right)$.

Assuming now $K_{\{1\}}(\mathrm{U}) \neq\{\}$, we let $\bar{U}:=\mathrm{U}_{\{1\}}$ and let $\overline{\bar{U}}$ consist of those rows of $\mathrm{U}$ whose first component is 0 , so we have $\overline{\bar{U}} \mathbf{e}_{1}=$ o. We assume w.l.o.g. that $U, \bar{U}$ are row-adjusted with $\kappa$, resp., $\bar{\kappa}$ blocks, cf. Definition 3.1, and that the rows of $\overline{\bar{U}}$ are partitioned as $\overline{\bar{U}}^{\top}=\left[\overline{\bar{U}}_{0}^{\top}|\ldots| \overline{\bar{U}}_{\kappa}^{\top}\right]=\overline{\bar{U}}_{[0: \kappa]}^{\top}$, such that the blocks of $\mathrm{U}$ additionally satisfy $\mathrm{U}_{k}=\left[\begin{array}{c}\overline{\mathrm{U}}_{k} \\ \overline{\mathrm{U}}_{k}\end{array}\right]$. We let $\bar{u}_{k}, \overline{\bar{u}}_{k}$, and $u_{k}=\bar{u}_{k}+\overline{\bar{u}}_{k}$ denote the numbers of rows of $\overline{\mathrm{U}}_{k}, \overline{\overline{\mathrm{U}}}_{k}$, and $\mathrm{U}_{k}$, for $k \in[0: \kappa]$, also allowing for $\bar{u}_{k}=0$ or $\overline{\bar{u}}_{k}=0$, corresponding to empty blocks $\overline{\mathrm{U}}_{k}, \overline{\overline{\mathrm{U}}}_{k}$. E.g., we have $\bar{u}_{k}=0$ for $\bar{\kappa}<k \leq \kappa$, and $\overline{\bar{u}}_{0}=0$, forced by $u_{0}=\bar{u}_{0}=1$. This also shows that the blocks $\overline{\bar{U}}_{k}$ do not constitute a row-adjusted representation of $\overline{\bar{U}}$, since $\operatorname{rank}^{(k)} \overline{\bar{U}}=\operatorname{rank}^{(k)} \overline{\bar{U}}_{[0: k]}$ does not hold in general, defining $\operatorname{rank}^{(k)} \mathrm{B}=0$ for $k \geq 0$, if $\mathrm{B}$ is an empty block. Still we have $\operatorname{rank}^{(k)} \overline{\overline{\mathrm{U}}}_{[0: k]}=\sum_{i=0}^{k} \overline{\bar{u}}_{i}$ for $k \in[0: \kappa]$, as well as $p_{\mathrm{U}}(x)-p_{\overline{\mathrm{U}}}(x)=\sum_{k=0}^{\kappa} \overline{\bar{u}}_{k} x^{k}$.

We make use of $\rho(\mathrm{W})=\rho(\widehat{W})$, where $\widehat{W}:=\left[\begin{array}{cc}\overline{\mathrm{U}} \otimes \boldsymbol{\eta}_{\ell} & \overline{\mathrm{U}} \mathbf{e}_{1} \otimes \mathrm{V} \\ \overline{\mathrm{U}} & \mathrm{O}\end{array}\right]$ is obtained from W by permuting columns and inserting an additional linearly dependent column $\mathbf{W}\left[\begin{array}{c}\boldsymbol{\eta}_{m} \\ \mathbf{o}\end{array}\right]$ in front. We define $\bar{W}:=$ $\left[\overline{\mathrm{U}} \otimes \boldsymbol{\eta}_{\ell} \mid \overline{\mathrm{U}} \mathbf{e}_{1} \otimes \mathrm{V}\right]$ and $\overline{\overline{\mathrm{W}}}:=[\overline{\overline{\mathrm{U}}} \mid \mathrm{O}]$. Using Remark 4.7, we observe $\operatorname{rank}^{(k)}(\overline{\mathrm{U}} \otimes \mathrm{V})=\operatorname{rank}^{(k)} \overline{\mathrm{W}}=$ $\operatorname{rank}^{(k)}\left[\overline{\mathbf{U}}_{[0: k]} \otimes \boldsymbol{\eta}_{\ell} \mid \overline{\mathbf{U}}_{[0: k]} \mathbf{e}_{1} \otimes \mathrm{V}\right]$, which, also employing the definition of blocks $\left(\overline{\mathbf{U}}_{i}\right)_{i \in[0, \kappa]}$, leads to

$$
\operatorname{rank}^{(k)}\left[\begin{array}{c}
\overline{\mathrm{W}} \\
\overline{\bar{W}}
\end{array}\right]=\operatorname{rank}^{(k)}\left[\begin{array}{cc}
\overline{\mathrm{U}}_{[0: k]} \otimes \boldsymbol{\eta}_{\ell} & \overline{\mathrm{U}}_{[0: k]} \mathbf{e}_{1} \otimes \mathrm{V} \\
\overline{\mathrm{U}}_{[0: k]} & \mathrm{O}
\end{array}\right]=\operatorname{rank}^{(k)} \overline{\mathrm{W}}+\operatorname{rank}^{(k)} \overline{\overline{\mathrm{U}}}_{[0: k]},
$$

the second equality following from $\operatorname{rowsp}\left(\overline{\mathrm{U}}_{[0: k]}^{\langle k\rangle}\right) \cap \operatorname{rowsp}\left(\overline{\bar{U}}_{[0: k]}^{\langle k\rangle}\right)=\left\{\mathbf{o}^{\top}\right\}$. Finally,

$$
\operatorname{rank}^{(k)} \widehat{\mathrm{W}}=\operatorname{rank}^{(k)}(\overline{\mathrm{U}} \otimes \mathrm{V})+\sum_{i=0}^{k} \overline{\bar{u}}_{i}, \quad \text { for } k \in[0: \kappa]
$$

and thus, $p_{\mathrm{W}}(x)=p_{\overline{\mathrm{U}}} \circledast \mathrm{V}(x)+\sum_{k=0}^{\kappa} \overline{\bar{u}}_{k} x^{k}=p_{\overline{\mathrm{U}}}(x) p_{\mathrm{V}}(x)+p_{\mathrm{U}}(x)-p_{\overline{\mathrm{U}}}(x)$. The lemma's last assertion now follows easily from Proposition $2.4 \mathrm{a}$ ) and properties of $p^{\star}$.

The next theorem generalizes Lemma 5.2.

THEOREM 5.3. Let $\mathrm{U}$ be a $k \times n$ matrix and let for $1 \leq i \leq n$ the matrices $\mathrm{V}_{i}$ be $k_{i} \times m_{i}$ matrices. If

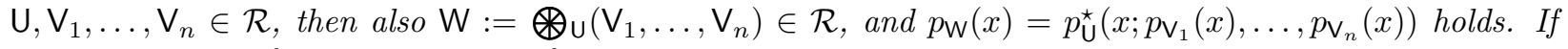
$\mathrm{U}, \mathrm{V}_{1}, \ldots, \mathrm{V}_{n} \in \mathcal{R}^{d}$, then also $\mathrm{W} \in \mathcal{R}^{d}$.

Proof. It is easily seen that $\mathrm{U}, \mathrm{V}_{1}, \ldots, \mathrm{V}_{n} \in \mathcal{R}$ implies $\mathrm{W} \in \mathcal{R}$, so we turn to $p_{\mathrm{W}}(x)$. We are going to 
construct $\mathrm{W}$ by replacing matrices $\mathrm{I}_{1}$ in $\mathrm{U}=\mathrm{U}_{\mathrm{U}}\left(\mathrm{I}_{1}, \ldots, \mathrm{I}_{1}\right)$ by $\mathrm{V}_{k}$ one by one, i.e., we consider

$$
\begin{aligned}
\mathrm{W}^{(n)} & :=\circledast \mathrm{U}(\underbrace{\mathrm{I}_{1}, \ldots, \mathrm{I}_{1}}_{n-1}, \mathrm{~V}_{n}), \\
\mathrm{W}^{(n-1)} & :=\mathrm{W}^{(n)}(\underbrace{\mathrm{I}_{1}, \ldots, \mathrm{I}_{1}}_{n-2}, \mathrm{~V}_{n-1}, \underbrace{\mathrm{I}_{1}, \ldots, \mathrm{I}_{1}}_{m_{n}})=\circledast \mathrm{U}(\underbrace{\mathrm{I}_{1}, \ldots, \mathrm{I}_{1}}_{n-2}, \mathrm{~V}_{n-1}, \mathrm{~V}_{n}),
\end{aligned}
$$

and generally, for $k \in[2: n]$,

$$
\mathrm{W}^{(k-1)}:=\circledast \mathrm{W}^{(k)}(\underbrace{\mathrm{I}_{1}, \ldots, \mathrm{I}_{1}}_{k-2}, \mathrm{~V}_{k-1}, \underbrace{\mathrm{I}_{1}, \ldots, \mathrm{I}_{1}}_{m_{k}+\cdots+m_{n}})=\circledast \mathrm{u}(\underbrace{\mathrm{I}_{1}, \ldots, \mathrm{I}_{1}}_{k-2}, \mathrm{~V}_{k-1}, \mathrm{~V}_{k}, \ldots, \mathrm{V}_{n}),
$$

with $\mathrm{W}=\mathrm{W}^{(1)}$ the result of all $n$ replacements. By Lemma 5.2, we have

$$
\begin{aligned}
p_{\mathrm{W}^{(k-1)}}(x) & =p_{\mathrm{W}^{(k)}}^{\star}(x ; \underbrace{1, \ldots, 1}_{k-2}, p_{\mathrm{V}_{k-1}}(x), \underbrace{1, \ldots, 1}_{m_{k}+\cdots+m_{n}}) \\
& =p_{\mathrm{W}^{(k)}}(x)+p_{\mathrm{W}_{\{k-1\}}^{(k)}}(x)\left(p_{\mathrm{V}_{k-1}}(x)-1\right) .
\end{aligned}
$$

We are now going to show by induction that for $k \in[1: n]$, we have

$$
p_{\mathrm{W}^{(k)}}(x)=\sum_{s \subseteq[k: n]} p_{\mathrm{U}_{s}}(x) \prod_{i \in s}\left(p_{\mathrm{V}_{i}}(x)-1\right),
$$

the case $k=1$ being the assertion of the theorem. Clearly, by Lemma 5.2, (5.5) is satisfied for $k=n$. Next, we assume that (5.5) has already been shown for all $k \in[\bar{k}: n]$, with $\bar{k} \geq 2$. We observe that $\mathrm{W}_{\{k-1\}}^{(k)}=\bigoplus_{\{k-1\}}(\underbrace{\mathrm{I}_{1}, \ldots, \mathrm{I}_{1}}_{k-1}, \mathrm{~V}_{k}, \ldots, \mathrm{V}_{n})$ holds, so equation (5.5) with $\mathrm{U}_{\{k-1\}}$ in place of $\mathrm{U}$ then also implies

$$
p_{\mathrm{W}_{\{k-1\}}^{(k)}}(x)=\sum_{s \subseteq[k: n]} p_{\mathrm{U}_{s \cup\{k-1\}}}(x) \prod_{i \in s}\left(p_{\mathrm{\vee}_{i}}(x)-1\right)
$$

for $k \in[\bar{k}: n]$. This leads to

$$
\begin{aligned}
p_{\mathrm{W}^{(\bar{k}-1)}}(x) & =p_{\mathrm{W}^{(\bar{k})}}(x)+p_{\mathrm{W}_{\{\bar{k}-1\}}^{(\bar{k})}}(x)\left(p_{\mathrm{\vee}_{\bar{k}-1}}(x)-1\right) \\
& =\sum_{s \subseteq[\bar{k}: n]} p_{\mathrm{U}_{s}}(x) \prod_{i \in s}\left(p_{\mathrm{\vee}_{i}}(x)-1\right)+\sum_{s \subseteq[\bar{k}: n]} p_{\mathrm{U}_{s \cup\{\bar{k}-1\}}}(x) \prod_{i \in s \cup\{\bar{k}-1\}}\left(p_{\mathrm{V}_{i}}(x)-1\right) \\
& =\sum_{s \subseteq[\bar{k}-1: n]} p_{\mathrm{\cup}_{s}}(x) \prod_{i \in s}\left(p_{\mathrm{\vee}_{i}}(x)-1\right),
\end{aligned}
$$

i.e., (5.5), and thus (5.6), also hold for $k=\bar{k}-1$, completing the proof by induction. Finally assume $\mathrm{U}, \mathrm{V}_{1}, \ldots, \mathrm{V}_{n} \in \mathcal{R}^{d}$. Decompose $\mathbf{x}=\left[\mathbf{x}_{1}^{\top}, \ldots, \mathbf{x}_{n}^{\top}\right]^{\top} \in \mathbb{R}^{m_{1}+\cdots+m_{n}}$ such that $\mathbf{x}_{i} \in \mathbb{R}^{m_{i}}$ for $1 \leq i \leq n$, and let $\mathbf{y}:=\left[\boldsymbol{\eta}_{m_{1}}^{\top} \mathbf{x}_{1}, \ldots, \boldsymbol{\eta}_{m_{n}}^{\top} \mathbf{x}_{n}\right]^{\top} \in \mathbb{R}^{n}$. Let $f_{\mathrm{U}}: \mathbb{R}^{n} \rightarrow \mathbb{R}, f_{\mathrm{V}_{i}}: \mathbb{R}^{m_{i}} \rightarrow \mathbb{R}, 1 \leq i \leq n$ be copositive forms of degree $d$, such that the rows of $\mathrm{U}$ are the zeros of $f_{\mathrm{U}}$ and the rows of $\mathrm{V}_{i}$ are the zeros of $f_{\mathrm{V}_{i}}$ for $1 \leq i \leq n$. Then the rows of $\mathrm{W}$ are all the zeros of $f_{\mathrm{W}}(\mathbf{x}):=f_{\mathrm{U}}(\mathbf{y})+\sum_{i=1}^{n} f_{\mathrm{V}_{i}}\left(\mathbf{x}_{i}\right)$, and clearly, $f_{\mathrm{W}}$ is a copositive form of degree d. This shows that $\mathrm{W} \in \mathcal{R}^{d}$.

REMARK 5.4. Define a partial order $\prec$ on polynomials $p, q \in \mathbb{R}[x]$, such that $q \prec p$ iff $p-q$ has nonnegative coefficients. Then $p_{i}, q_{i} \in 1+x \mathbb{R}[x], 0 \prec q_{i} \prec p_{i}$ for $i \in[1: n]$ implies $p_{\mathrm{U}}^{\star}\left(\cdot ; q_{1}, \ldots, q_{n}\right) \prec$ $p_{\mathrm{U}}^{\star}\left(\cdot ; p_{1}, \ldots, p_{n}\right)$ for $\mathrm{U}$ such as in Theorem 5.3. 
Electronic Journal of Linear Algebra, ISSN 1081-3810

A publication of the International Linear Algebra Society

Volume 36, pp. 519-541, August 2020.

6. Completely positive forms of degree $\geq 3$. We will first present copositive forms of degree 3 and 4 having zero sets that allow for rather direct computation of the generating polynomials. Copositive forms of degree larger than 4 having those same two zero sets are easily obtained. Extracting coefficients then leads to Theorem 6.3, stating a lower bound for $p_{N}^{(d)}$ that asymptotically matches the upper bound, derived from Carathéodory's theorem.

For later use, in the following lemma we derive the rank differences generating polynomials of some particular matrices with entries in $\{-1,1\}$.

Lemma 6.1. Let, for $N \geq 2$, the row set of $\mathrm{B}_{N} \in \mathbb{R}^{2^{N-1} \times N}$ be the set $\{1\} \times\{-1,1\}^{N-1}$. Then we have $p_{\mathrm{B}_{N}}(x)=(1+x)^{N-1}$.

Proof. We may and do assume that the rows of $\mathrm{B}_{N}$ are colexicographically ordered. Then $\mathrm{B}_{2}=\left(\begin{array}{cc}1 & -1 \\ 1 & 1\end{array}\right)$ has full rank 2 , and therefore $p_{\mathrm{B}_{2}}(x)=1+x$, so the assertion of the lemma is true for $N=2$. By Remark $3.6 \mathrm{~b}$ ) we then have $p_{\mathrm{B}_{2}}^{\star}\left(x ; z_{1}, z_{2}\right)=(1+x) z_{1} z_{2}$. Next we observe

$$
\mathrm{B}_{3}=\left[\begin{array}{ccc}
1 & -1 & -1 \\
1 & 1 & -1 \\
1 & -1 & 1 \\
1 & 1 & 1
\end{array}\right]=\circledast_{\left[\begin{array}{cc}
1 & -1 \\
1 & 1
\end{array}\right]}\left(\left[\begin{array}{cc}
1 & -1 \\
1 & 1
\end{array}\right], 1\right)=\bigotimes_{\mathrm{B}_{2}}\left(\mathrm{~B}_{2}, 1\right)
$$

More generally, we have $\mathrm{B}_{N+1}=\otimes_{\mathrm{B}_{2}}\left(\mathrm{~B}_{N}, 1\right)$ for $N \geq 2$, leading to

$$
p_{\mathrm{B}_{N+1}}(x)=p_{\mathrm{B}_{2}}^{\star}\left(x ; p_{\mathrm{B}_{N}}(x), 1\right)=(1+x)(1+x)^{N-1}=(1+x)^{N},
$$

which completes the proof by induction.

Proposition 6.2. For any $N \in \mathbb{N}$, there exist matrices $\mathrm{A}_{N} \in \mathcal{R}^{3}$ and $\mathrm{A}_{N}^{\prime} \in \mathcal{R}^{4}$ with $N$ columns, with all entries of $\mathrm{A}_{N}^{\prime}$ strictly positive, such that $p_{\mathrm{A}_{N}}(x)=p_{\mathrm{A}_{N}^{\prime}}(x)=(1+x)^{N-1}$ holds.

Proof. For $N=1$, we may choose $\mathrm{A}_{1}=\mathrm{A}_{1}^{\prime}=1$, yielding $p_{\mathrm{A}_{1}}(x)=p_{\mathrm{A}_{1}^{\prime}}(x)=1$, and we may thus assume $N \geq 2$. Note that

$$
f\left(x_{1}, \ldots, x_{N}\right):=\sum_{i=2}^{N} x_{i}\left(N x_{i}-\sum_{j=1}^{N} x_{j}\right)^{2}
$$

is a copositive form of degree 3 with zero set

$$
\mathcal{Z}_{N}=\left\{\mathbf{x} \in \Delta^{N}: x_{i} \in\left\{0, \frac{1}{N}\right\} \text { for } 2 \leq i \leq N\right\}
$$

We have $\left|\mathcal{Z}_{N}\right|=2^{N-1}$, and define the matrix $A_{N}$ with row set equal to $\mathcal{Z}_{N}$. As in the proof of Lemma 6.1 , we assume that the rows of $\mathrm{A}_{N}$ are colexicographically ordered, and with the matrix $\mathrm{B}_{N}$ from that proof, we have

$$
\mathrm{B}_{N}=\mathrm{A}_{N} \mathrm{Q}_{N}
$$

where $Q_{N}=\left(q_{i, j}\right)_{1 \leq i, j \leq N}$ satisfies $q_{i, 1}=1$ for $i \in[1: N], q_{i, i}=2 N-1$ for $i \in[2: N]$, and $q_{i, j}=-1$ in all other cases. For example, for $N=3$ we have

$$
\mathrm{A}_{3} \mathrm{Q}_{3}=\frac{1}{3}\left[\begin{array}{lll}
3 & 0 & 0 \\
2 & 1 & 0 \\
2 & 0 & 1 \\
1 & 1 & 1
\end{array}\right]\left[\begin{array}{ccc}
1 & -1 & -1 \\
1 & 5 & -1 \\
1 & -1 & 5
\end{array}\right]=\left[\begin{array}{ccc}
1 & -1 & -1 \\
1 & 1 & -1 \\
1 & -1 & 1 \\
1 & 1 & 1
\end{array}\right]=B_{3}
$$


Now $\mathrm{Q}_{N}$ has full rank because of $\operatorname{det} \mathrm{Q}_{N}=(2 N)^{N-1}$, so $p_{\mathrm{A}_{N}}(x)=p_{\mathrm{B}_{N}}(x)=(1+x)^{N-1}$ by Proposition 2.4 and Lemma 6.1. For the construction of $\mathrm{A}_{N}^{\prime}$, we use

$$
g\left(x_{1}, \ldots, x_{N}\right):=\sum_{i=1}^{N-1}\left(\left(\begin{array}{c}
N+1 \\
2
\end{array}\right)^{2}\left(x_{i+1}-x_{i}\right)^{2}-\left(\sum_{j=1}^{N} x_{j}\right)^{2}\right)^{2}
$$

which is a copositive form of degree 4 with zero set

$$
\mathcal{Z}_{N}^{\prime}=\left\{\mathrm{x} \in \Delta^{N}:\left|x_{i+1}-x_{i}\right|=\frac{2}{N(N+1)} \text { for } 1 \leq i<N\right\}
$$

completely contained in the relative interior of $\Delta^{N}$. Denoting $\delta_{N}=\frac{2}{N(N+1)}$, it is quite easy to check that $\mathbf{x} \in \mathcal{Z}_{N}^{\prime}$ implies $x_{i} \geq \delta_{N}$ for all $i \in[1: N]$. (Assuming $x_{i}<\delta_{N}$, note $x_{j}=x_{i}+\left(x_{j}-x_{i}\right) \leq x_{i}+|j-i| \delta_{N}<$ $(|j-i|+1) \delta_{N}$ for all $j \in[1: N]$. This results in

$$
1=\sum_{j=1}^{N} x_{j}<\delta_{N}+\sum_{j=2}^{i} j \delta_{N}+\sum_{j=2}^{N-i+1} j \delta_{N} \leq \delta_{N} \sum_{j=1}^{N} j=\left(\begin{array}{c}
N+1 \\
2
\end{array}\right) \delta_{N}=1
$$

a contradiction.) Now define $\mathrm{A}_{N}^{\prime}$ composed of rows $\mathbf{x} \in \mathcal{Z}_{N}^{\prime}$, such that their first differences $\Delta \mathbf{x}:=\left(x_{2}-\right.$ $\left.x_{1}, x_{3}-x_{2}, \ldots, x_{N}-x_{N-1}\right) \in\left\{-\delta_{N}, \delta_{N}\right\}^{N-1}$ are colexicographically ordered. For example,

$$
\mathrm{A}_{3}^{\prime}=\frac{1}{18}\left[\begin{array}{lll}
9 & 6 & 3 \\
5 & 8 & 5 \\
7 & 4 & 7 \\
3 & 6 & 9
\end{array}\right]
$$

because ( $u p$ to factors $\delta_{3}=\frac{1}{6}$ ) we have $(-1,-1)<(1,-1)<(-1,1)<(1,1)$. Again, there is a simple correspondence between matrices $\mathrm{A}_{N}^{\prime}$ and matrices $\mathrm{B}_{N}$ from Lemma 6.1, namely $\mathrm{B}_{N}=\mathrm{A}_{N}^{\prime} \mathrm{Q}_{N}^{\prime}$, where $\mathrm{Q}_{N}^{\prime}=\left(q_{i, j}^{\prime}\right)$ satisfies $q_{i, 1}^{\prime}=1$ for $i \in[1: N], q_{i, i+1}^{\prime}=-\left(\begin{array}{c}N+1 \\ 2\end{array}\right)$ for $i \in[1: N-1], q_{i, i}^{\prime}=\left(\begin{array}{c}N+1 \\ 2\end{array}\right)$ for $i \in[2: N]$, and $q_{i, j}^{\prime}=0$ in all other cases. E.g., for $N=3$, we have

$$
\mathrm{A}_{3}^{\prime} \mathrm{Q}_{3}^{\prime}=\frac{1}{18}\left[\begin{array}{lll}
9 & 6 & 3 \\
5 & 8 & 5 \\
7 & 4 & 7 \\
3 & 6 & 9
\end{array}\right]\left[\begin{array}{ccc}
1 & -6 & 0 \\
1 & 6 & -6 \\
1 & 0 & 6
\end{array}\right]=\left[\begin{array}{ccc}
1 & -1 & -1 \\
1 & 1 & -1 \\
1 & -1 & 1 \\
1 & 1 & 1
\end{array}\right]=\mathrm{B}_{3}
$$

It is an easy exercise to show $\operatorname{det} \mathrm{Q}_{N}^{\prime}=N\left(\begin{array}{c}N+1 \\ 2\end{array}\right)^{N-1}$, so $\mathrm{Q}_{N}^{\prime}$ has full rank, and the proof continues as before.

TheOrem 6.3. Let $d \in \mathbb{N}$ satisfy $d \geq 3$. Then

$$
\left(\begin{array}{l}
N \\
d
\end{array}\right)<\sum_{i=0}^{d}\left(\begin{array}{c}
N-1 \\
i
\end{array}\right) \leq p_{N}^{(d)} \leq\left(\begin{array}{c}
N+d-1 \\
d
\end{array}\right)
$$

i.e., $p_{N}^{(d)}=\left(\begin{array}{l}N \\ d\end{array}\right)\left(1+\mathcal{O}\left(\frac{d^{2}}{N}\right)\right)$, uniformly in $d$, as $N \rightarrow \infty$.

Proof. By Remark 5.1, the matrix $\mathrm{A}_{N}$ defined in Proposition 6.2 satisfies $\mathrm{A}_{N} \in \mathcal{R}^{d}$. From $p_{\mathrm{A}_{N}}(x)=$ $(1+x)^{N-1}$, the left hand inequality

$$
p_{N}^{(d)} \geq \operatorname{rank}^{(d)} \mathrm{A}_{N}=\sum_{i=0}^{d}\left(\begin{array}{c}
N-1 \\
i
\end{array}\right) \geq\left(\begin{array}{c}
N-1 \\
d
\end{array}\right)+\left(\begin{array}{c}
N-1 \\
d-1
\end{array}\right)=\left(\begin{array}{c}
N \\
d
\end{array}\right)
$$


follows via Corollary 2.2. The right hand inequality follows from Carathéodory's theorem, see also Corollary 2.2. Finally, from $\left(\begin{array}{c}N+d-1 \\ d\end{array}\right)-\left(\begin{array}{l}N \\ d\end{array}\right) \leq(e-1) \frac{d^{2}}{N}\left(\begin{array}{l}N \\ d\end{array}\right)$, holding for fixed $d$, if $N$ is large enough, we see that we have derived asymptotically matching lower and upper bounds, and the last assertion follows. We prove the latter inequality by restricting our attention to $N=d^{2}+d-1+m$ with $m \geq 0$, for which we observe

$$
\begin{aligned}
\left(\begin{array}{c}
N+d-1 \\
d
\end{array}\right) /\left(\begin{array}{l}
N \\
d
\end{array}\right) & =\prod_{k=0}^{d-1}\left(1+\frac{d-1}{d^{2}+m+k}\right) \leq\left(1+\frac{d-1}{d^{2}+m}\right)^{d} \\
& \leq \exp \left(\frac{d(d-1)}{d^{2}+m}\right) \leq 1+(e-1) \frac{d(d-1)}{d^{2}+m} \leq 1+(e-1) \frac{d^{2}}{N}
\end{aligned}
$$

using $1+x \leq e^{x} \leq 1+(e-1) x$ for $0 \leq x \leq 1$.

7. An improved lower bound for the cp-rank of completely positive matrices. We will construct a sequence $\left(\mathcal{J}^{(k)}\right)_{k \geq 1}$ of sets of matrices, each set $\mathcal{J}^{(k)}=\left\{\mathrm{J}_{N}^{(k)}: N \geq 1\right\}$ containing one matrix of each order $N$, starting with $\mathcal{J}^{(\overline{1})}=\left\{\mathrm{I}_{N}: N \geq 1\right\}$. We then use matrices as considered in Theorem 3.7 together with matrices from $\mathcal{J}^{(k)}$ in the construction introduced in Section 5 to build matrices of the set $\mathcal{J}^{(k+1)}$, keeping one matrix of each order, that achieves a high 2-rank. A lower bound for the maximal 2-rank achieved by the order $N$ matrices in $\bigcup_{k \geq 1} \mathcal{J}^{(k)}$, yielding a lower bound for $p_{N}$, will be presented in Theorem 7.4. Choosing members of $\mathcal{J}^{(k)}$ optimally is possible with computer assistance for small orders, and some results will be presented in Table 1. We start with two Lemmas.

LEMmA 7.1. Let $f(x)=\frac{x^{2}}{2}+C N\left(\frac{N}{x}\right)^{\alpha}$ be defined on $] 0, \infty\left[\right.$, with $C, \alpha, N>0$ satisfying $C \alpha N^{1+\alpha}>$ $3^{2+\alpha}$. Then

$$
f^{*}:=\min _{\substack{n \geq 5 \\ n \text { odd }}} f(n) \leq(C \alpha)^{\frac{2}{2+\alpha}}\left(\frac{1}{2}+\frac{1}{\alpha}\right) N^{\frac{2+2 \alpha}{2+\alpha}}+4+2 \alpha .
$$

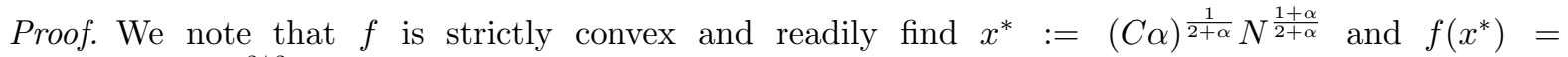
$(C \alpha)^{\frac{2}{2+\alpha}}\left(\frac{1}{2}+\frac{1}{\alpha}\right) N^{\frac{2+2 \alpha}{2+\alpha}}$, the minimum of $f$ among the positive reals, where the condition on $C, \alpha, N$ is equivalent to $x^{*}>3$. Defining $\kappa:=2\left[\frac{x^{*}-1}{2}\right]+1$, we have $\max \left(5, x^{*}\right) \leq \kappa \leq x^{*}+2$, and thus, $f^{*} \leq f(\kappa)$. By Taylor's Theorem,

$$
f(\kappa)=f\left(x^{*}\right)+f(\kappa)-f\left(x^{*}\right) \leq f\left(x^{*}\right)+\frac{\left(\kappa-x^{*}\right)^{2}}{2 !} \max _{x^{*} \leq x \leq \kappa} f^{\prime \prime}(x) \leq f\left(x^{*}\right)+2(2+\alpha),
$$

where we used $\left|\kappa-x^{*}\right| \leq 2$, monotonicity of $f^{\prime \prime}(x)=1+C \alpha(\alpha+1) N^{1+\alpha} x^{-2-\alpha}$, and $f^{\prime \prime}\left(x^{*}\right)=2+\alpha$.

LEMmA 7.2. Let $N, n, \ell \in \mathbb{N}$ satisfy $N=m(\ell+1)+(n-m) \ell=n \ell+m$ with $0 \leq m<n$. Then, for $0 \leq \alpha \leq 1$,

$$
m(\ell+1)^{1+\alpha}+(n-m) \ell^{1+\alpha} \leq \frac{N^{1+\alpha}}{n^{\alpha}}+\frac{\alpha}{4} N
$$

Proof. By some straightforward manipulations, we have

$$
m(\ell+1)^{2}+(n-m) \ell^{2}=\frac{n^{2} \ell^{2}+2 n \ell m+m^{2}+m(n-m)}{n}=\frac{N^{2}}{n}+\frac{m(n-m)}{n},
$$

with $\frac{m(n-m)}{n} \leq \frac{n}{4}$. Then, for $\alpha \in[0,1]$ we use Jensen's and Bernoulli's inequalities, together with (7.7), to 
obtain

$$
\begin{aligned}
m(\ell+1)^{1+\alpha}+(n-m) \ell^{1+\alpha} & =N\left(\frac{m(\ell+1)}{N}(\ell+1)^{\alpha}+\frac{(n-m) \ell}{N} \ell^{\alpha}\right) \\
& \leq N\left(\frac{m(\ell+1)}{N}(\ell+1)+\frac{(n-m) \ell}{N} \ell\right)^{\alpha}=N\left(\frac{N}{n}+\frac{m(n-m)}{N n}\right)^{\alpha} \\
& \leq N\left(\frac{N}{n}+\frac{n}{4 N}\right)^{\alpha} \leq \frac{N^{1+\alpha}}{n^{\alpha}}\left(1+\frac{\alpha}{4} \frac{n^{2}}{N^{2}}\right) \leq \frac{N^{1+\alpha}}{n^{\alpha}}+\frac{\alpha}{4} N .
\end{aligned}
$$

Proposition 7.3. Define constants $\alpha_{k}:=\frac{1}{2^{k}-1}, C_{k}:=\left(1-2^{-k}\right) 2^{1-k \alpha_{k}}$ for $k \in \mathbb{N}$, and $d_{k}:=2 k-1-$ $\alpha_{k-1}$ for $k \geq 2$, and $d_{1}:=-1$. Then, for any $k, N \in \mathbb{N}$ there is a matrix $\mathrm{J}_{N}^{(k)} \in \mathcal{R}^{2}$ with $N$ columns, such that

$$
\begin{aligned}
\operatorname{rank} J_{N}^{(k)} & =N \\
\operatorname{rank}^{(2)} J_{N}^{(k)} & \geq \frac{N^{2}}{2}-C_{k} N^{1+\alpha_{k}}-d_{k} N
\end{aligned}
$$

hold.

Proof. We start defining $\mathrm{J}_{N}^{(1)}:=\mathrm{I}_{N}$ for $N \geq 1$ (here $C_{1}=\frac{1}{2}, \alpha_{1}=1,(7.8)$ is satisfied, and (7.9) is actually an equality). The construction continues inductively. Having defined $\left(\mathrm{J}_{N}^{(k)}\right)_{N \geq 1}$ for some $k \geq 1$, we are going to define $\mathrm{J}_{N}^{(k+1)}:=\mathrm{J}_{N}^{(k)}$ for $N$ small, and $\mathrm{J}_{N}^{(k+1)}$ equal to

$$
\mathrm{W}_{N, n}:=\circledast \mathrm{U}_{n}(\underbrace{\mathrm{J}_{\ell+1}^{(k)}, \ldots, \mathrm{J}_{\ell+1}^{(k)}}_{m \text { times }}, \underbrace{\mathrm{J}_{\ell}^{(k)}, \ldots, \mathrm{J}_{\ell}^{(k)}}_{n-m \text { times }}),
$$

with carefully chosen $n \in[5: N], \ell \in \mathbb{N}$ and $0 \leq m<n$, and $\mathrm{U}_{n}$ as in Theorem 3.7, for $N=n \ell+m$ large.

We first deal with small $N$. Define $\nu_{1}:=8$ and $\nu_{k}:=\left\lfloor\varphi^{2^{k+1}-1}\right\rfloor$ for $k \geq 2$, with $\varphi=\frac{1+\sqrt{5}}{2}$. We want to show that for $k \geq 1, N \leq \nu_{k}$ we have

$$
C_{k} N^{\alpha_{k}}-C_{k+1} N^{\alpha_{k+1}} \leq d_{k+1}-d_{k} .
$$

For $k=1$, we see that $C_{1} N^{\alpha_{1}}-C_{2} N^{\alpha_{2}}=\frac{1}{2} N-\frac{3}{2^{5 / 3}} N^{\frac{1}{3}}<3=d_{2}-d_{1}$ holds for $N \leq \nu_{1}$. Let now $k \geq 2$. Using $\varphi^{2}-\varphi=1$, we have

$$
\begin{aligned}
N \leq \nu_{k} & \Longrightarrow N^{\alpha_{k+1}} \leq \varphi \Longrightarrow N^{2 \alpha_{k+1}}-N^{\alpha_{k+1}} \leq 1 \\
& \Longrightarrow C_{k} N^{\alpha_{k}}-C_{k+1} N^{\alpha_{k+1}} \leq C_{k} N^{2 \alpha_{k+1}}-C_{k} N^{\alpha_{k+1}} \leq C_{k}<2 \leq d_{k+1}-d_{k},
\end{aligned}
$$

which yields (7.11). The first inequality in the second line follows from

$$
\begin{aligned}
N^{\alpha_{k+1}}\left(N^{\alpha_{k}-2 \alpha_{k+1}}-1\right) & \leq \varphi\left(\varphi^{\alpha_{k}}-1\right) \leq 3 \varphi\left(\varphi^{\frac{1}{3}}-1\right) \alpha_{k} \leq \frac{1}{2}(1+(k-1) \log 2) \alpha_{k} \\
& \leq\left(1+\frac{\alpha_{k}}{2}\right) 2^{k \alpha_{k}-(k+1) \alpha_{k+1}}-1=\frac{C_{k+1}}{C_{k}}-1 .
\end{aligned}
$$

In the latter chain of four inequalities, we used correspondingly:

(i) $\alpha_{k}-2 \alpha_{k+1}=\alpha_{k} \alpha_{k+1}$ (this is also used in (iv) below),

(ii) monotonicity of $\frac{\varphi^{\alpha}-1}{\alpha}$ for $\alpha>0$ : $\frac{\varphi^{\alpha} k-1}{\alpha_{k}} \leq \frac{\varphi^{\alpha_{2}}-1}{\alpha_{2}}=3\left(\varphi^{\frac{1}{3}}-1\right)$, 
(iii) the $k=2$ case: $3 \varphi\left(\varphi^{\frac{1}{3}}-1\right)=0.84454 \ldots<0.84657 \ldots=\frac{1}{2}(1+\log 2)$, and

(iv) the inequality $a+b \leq(1+a) e^{b} 2^{c}-1$, valid for $a, b, c \geq 0$, where $a=\frac{\alpha_{k}}{2}, b=\frac{k-1}{2} \alpha_{k} \log 2$ and $c=\frac{k+1}{2} \alpha_{k} \alpha_{k+1}$, and finally $\frac{k-1}{2} \alpha_{k}+c=k \alpha_{k}-(k+1) \alpha_{k+1}$.

Therefore, for $k \geq 1$, we define $\mathrm{J}_{N}^{(k+1)}:=\mathrm{J}_{N}^{(k)}$ for $N \leq \nu_{k}$, yielding

$$
\operatorname{rank}^{(2)} \mathrm{J}_{N}^{(k+1)} \geq \frac{N^{2}}{2}-C_{k} N^{1+\alpha_{k}}-d_{k} N \geq \frac{N^{2}}{2}-C_{k+1} N^{1+\alpha_{k+1}}-d_{k+1} N
$$

by (7.11), in accordance with (7.8) and (7.9).

Next, we assume $N>\nu_{k}$. Using Theorem 5.3, Theorem 3.7 d), Corollary 3.9 with

$$
\begin{aligned}
& a=\ell+\left(\frac{(\ell+1)^{2}}{2}-C_{k}(\ell+1)^{1+\alpha_{k}}-\left(d_{k}+1\right)(\ell+1)\right) x, \\
& b=\ell-1+\left(\frac{\ell^{2}}{2}-C_{k} \ell^{1+\alpha_{k}}-\left(d_{k}+1\right) \ell\right) x,
\end{aligned}
$$

where $1+a x \prec p_{\mathbf{J}_{\ell+1}^{(k)}}(x), 1+b x \prec p_{\mathbf{J}_{\ell}^{(k)}}(x)$ by assumption, and Remark 5.4, we find

$$
\begin{aligned}
p_{\mathrm{W}_{N, n}}(x) \succ & +(n-2) x+x^{\epsilon_{n}}+(1+(n-3) x)(m \ell+(n-m)(\ell-1)) x \\
& +m\left(\frac{(\ell+1)^{2}}{2}-C_{k}(\ell+1)^{1+\alpha_{k}}-\left(d_{k}+1\right)(\ell+1)\right) x^{2} \\
& +(n-m)\left(\frac{\ell^{2}}{2}-C_{k} \ell^{1+\alpha_{k}}-\left(d_{k}+1\right) \ell\right) x^{2} \\
& -\frac{1}{2}\left(m \ell^{2}+(n-m)(\ell-1)^{2}\right) x^{2}+\frac{1}{2}(m \ell+(n-m)(\ell-1))^{2} x^{2}+\mathcal{O}\left(x^{3}\right) .
\end{aligned}
$$

The identities $m \ell+(n-m)(\ell-1)=N-n$ and $m \frac{(\ell+1)^{2}-\ell^{2}}{2}+(n-m) \frac{\ell^{2}-(\ell-1)^{2}}{2}=N-\frac{n}{2}$ lead to rank $\mathrm{W}_{N, n}=$ $N+1-\epsilon_{n}$. So, we have rank $\mathrm{W}_{N, n}=N$ iff $n$ is odd, which we from now on assume. Furthermore,

$$
\begin{aligned}
\operatorname{rank}^{(2)} \mathrm{W}_{N, n} \geq & N+(n-3)(N-n)+\frac{1}{2}(N-n)^{2}+N-\frac{n}{2}-\left(d_{k}+1\right) N \\
& -C_{k}\left(m(\ell+1)^{1+\alpha_{k}}+(n-m) \ell^{1+\alpha_{k}}\right) \\
\geq & \frac{N^{2}}{2}-\frac{n^{2}}{2}-\left(2+d_{k}\right) N+\frac{5}{2} n-C_{k}\left(\frac{N^{1+\alpha_{k}}}{n^{\alpha_{k}}}+\frac{\alpha_{k}}{4} N\right),
\end{aligned}
$$

where we employed Lemma 7.2. Note that $N>\nu_{k}$ implies $C_{k} \alpha_{k} N^{1+\alpha_{k}}>3^{2+\alpha_{k}}$. This is easily checked for $k=1$. For $k \geq 2$, note that, by the Mean Value Theorem, we have $2^{k+1}-2^{2+1} \geq(k-2) 8 \log 2 \geq(k-2) \frac{\log 2}{\log \varphi}$, implying $\varphi^{2^{k+1}-1} \geq \varphi^{7} 2^{k-2}$. Next, for $N \geq \nu_{k}+1>\varphi^{2^{k+1}-1}$ we deduce the claimed inequality from

$$
C_{k} \alpha_{k} N^{1+\alpha_{k}} 3^{-2-\alpha_{k}}=\frac{2}{3}\left(\frac{N}{3 \cdot 2^{k}}\right)^{1+\alpha_{k}}>\frac{2}{3}\left(\frac{\varphi^{7}}{12}\right)^{1+\alpha_{k}}>\frac{2}{3} \frac{\varphi^{7}}{12}>1,
$$

so the application of Lemma 7.1 is justified. Now, by fixing $\bar{n} \geq 5$ to be the smallest odd number larger than $\left(C_{k} \alpha_{k}\right)^{\frac{1}{2+\alpha_{k}}} N^{\frac{1+\alpha_{k}}{2+\alpha_{k}}}$, and invoking Lemma 7.1, noting that $\frac{2+2 \alpha_{k}}{2+\alpha_{k}}=1+\alpha_{k+1}$ and $\left(C_{k} \alpha_{k}\right)^{\frac{2}{2+\alpha_{k}}}\left(\frac{1}{2}+\frac{1}{\alpha_{k}}\right)=$ $\left(2^{1-k\left(1+\alpha_{k}\right)}\right)^{1-\alpha_{k+1}} \frac{1}{2 \alpha_{k+1}}=C_{k+1}$, we further obtain

$$
\begin{aligned}
\operatorname{rank}^{(2)} \mathrm{W}_{N, \bar{n}} & \geq \frac{N^{2}}{2}-\left(2+d_{k}+\frac{1}{4} C_{k} \alpha_{k}\right) N+\frac{5}{2} \bar{n}-C_{k+1} N^{1+\alpha_{k+1}}-4-2 \alpha_{k} \\
& >\frac{N^{2}}{2}-C_{k+1} N^{1+\alpha_{k+1}}-d_{k+1} N
\end{aligned}
$$


where the last inequality uses the definition of $d_{k}$, the inequalities $\frac{C_{k}}{4}<\frac{1}{2}$ and $\frac{3}{2} \alpha_{k}<\alpha_{k-1}$, and furthermore, $\frac{5}{2} \bar{n}-4-2 \alpha_{k} \geq 0$ for $\bar{n} \geq 5$. Defining $\mathrm{J}_{N}^{(k+1)}:=\mathrm{W}_{N, \bar{n}}$ completes the construction, with both (7.8) and (7.9) satisfied.

THEOREM 7.4. For $N \geq 1$, we have

$$
p_{N} \geq \frac{N^{2}}{2}-\left(2 \log _{2} \log N+e^{2}\right) N
$$

Proof. From (7.9), we deduce $p_{N} \geq \frac{N^{2}}{2}-2 N^{1+\alpha_{k}}-(2 k-2) N$ for any $k \in \mathbb{N}$, using Corollary 2.2. Fixing $k:=\left\lceil\log _{2} \log N\right\rceil$ leads to $2 k-2 \leq 2 \log _{2} \log N$ and

$$
k \geq \log _{2} \log N \Longrightarrow \alpha_{k} \leq \frac{1}{\log N-1} \Longrightarrow N^{\alpha_{k}} \leq \exp \left(\frac{\log N}{\log N-1}\right) \leq \frac{e^{2}}{2}
$$

for $N \geq 71>\exp \left(\frac{2-\log 2}{1-\log 2}\right)$. For $N \in[1: 70]$, we numerically check that the right hand side of $(7.12)$ is smaller than the lower bound $s_{N}-N\left(\sqrt{2 N}-\frac{3}{2}\right)$ given in (1.1).

In order to keep the proofs of Proposition 7.3 and Theorem 7.4 simple, we decided for rather crude lower estimates at some places at the risk of having not the best possible constants in Theorem 7.4. To illustrate what can be gained with some more effort, we consider $N=50$ in some detail. The estimate from Theorem 7.4 is $p_{50} \geq\left\lceil\frac{50^{2}}{2}-\left(2 \log _{2} \log 50+e^{2}\right) 50\right\rceil=684$, which is just a bit larger than the Drew-JohnsonLoewy lower bound $\left\lfloor\frac{50^{2}}{4}\right\rfloor=625$. Now assume that we employ a more straightforward, yet harder to analyse, recursive construction than that in Proposition 7.3, with $\mathbf{W}_{N, n}$ as in (7.10),

$$
\begin{aligned}
& \overline{\mathrm{J}}_{N}^{(1)}:=\mathrm{I}_{N}, \text { for } N \in \mathbb{N} \\
& \overline{\mathrm{J}}_{N}^{(k+1)} \in \arg \max \left\{\operatorname{rank}^{(2)} \mathrm{M}: \mathrm{M} \in\left\{\overline{\mathrm{J}}_{N}^{(k)}\right\} \cup\left\{\mathrm{W}_{N, n}: n \text { odd, } n \in[5: N]\right\}\right\}, \text { for } k, N \in \mathbb{N} .
\end{aligned}
$$

That is, we have $\bar{J}_{N}^{(k+1)}:=\mathrm{W}_{N, n^{*}}$ in the large $N$ case, where $n^{*}=n^{*}(k, N)$ maximizes $\operatorname{rank}^{(2)} \mathrm{W}_{N, n}$ from (7.10), rather than just being an approximate maximizer of a lower bound of $\operatorname{rank}^{(2)} \mathrm{W}_{N, n}$, as in the proof of Proposition 7.3. Note that the construction is not sensitive to the choice of a particular element from a non-singleton arg max.

With computer assistance, these discrete optimization problems can be easily solved for small $k, N$. See Table 1 for some results on $\bar{b}_{N}:=\max _{k \geq 1} \operatorname{rank}^{(2)} \overline{\mathrm{J}}_{N}^{(k)}$, with $\bar{k}=\bar{k}(N):=\min \left\{k: \bar{b}_{N}=\operatorname{rank}^{(2)} \overline{\mathrm{J}}_{N}^{(k)}\right\}$, and Figure 1 for a comparison of lower bounds, which suggests that with $\bar{b}_{N}$ we just gain an improvement of the constant $e^{2}$ in (7.12). The table includes in particular entries for those $N$ where $\bar{k}(N)$ has its first four jumps. In the table, the matrix encoded by $a_{m} b_{n-m}$, with $m \in[0: n-1]$, is the matrix $\bigotimes_{n}(\underbrace{\overline{\mathrm{J}}_{a}^{(\bar{k})}, \ldots, \overline{\mathrm{J}}_{a}^{(\bar{k})}}_{m \text { times }}, \underbrace{\overline{\mathrm{J}}_{b}^{(\bar{k})}, \ldots, \overline{\mathrm{J}}_{b}^{(\bar{k})}}_{n-m})$. Note that the arg max above has indeed sometimes cardinality greater than one: Both matrices $4_{5} 3_{2}$ and $3_{8} 2_{1}$ lead to the same bound $\bar{b}_{26}=256$, further examples being the pairs $\left(5_{3} 4_{6}, 4_{6} 3_{5}\right)$ and $\left(5_{5} 4_{6}, 7_{7}\right)$. Returning now to $N=50$, we obtain $\overline{\mathrm{J}}_{50}^{(3)}=\mathrm{u}_{7}\left(\overline{\mathrm{J}}_{8}^{(2)}, \overline{\mathrm{J}}_{7}^{(2)}, \overline{\mathrm{J}}_{7}^{(2)}, \overline{\mathrm{J}}_{7}^{(2)}, \overline{\mathrm{J}}_{7}^{(2)}, \overline{\mathrm{J}}_{7}^{(2)}, \overline{\mathrm{J}}_{7}^{(2)}\right)$, with $\overline{\mathrm{J}}_{7}^{(2)}=\mathrm{u}_{5}\left(\mathrm{I}_{2}, \mathrm{I}_{2}, \mathrm{I}_{1}, \mathrm{I}_{1}, \mathrm{I}_{1}\right)$ and $\bar{J}_{8}^{(2)}=\mathrm{U}_{5}\left(\mathrm{I}_{2}, \mathrm{I}_{2}, \mathrm{I}_{2}, \mathrm{I}_{1}, \mathrm{I}_{1}\right)$. These matrices have generating polynomials $p_{\overline{\mathrm{J}}_{7}^{(2)}}(x)=1+6 x+5 x^{2}+x^{3}$, $p_{\bar{J}_{8}^{(2)}}(x)=1+7 x+9 x^{2}+3 x^{3}$, and $p_{\bar{J}_{50}^{(3)}}(x)=1+49 x+1003 x^{2}+\mathcal{O}\left(x^{3}\right)$, leading to $p_{50} \geq \bar{b}_{50}:=1053$. Note that the lower bounds derived from $\bar{J}_{7}^{(2)}$ and $\bar{J}_{8}^{(2)}$ just listed are worse than the lower bounds $p_{7} \geq 14, p_{8} \geq 18$ derived in [9, Examples 1 and 3] from ad hoc constructed matrices. 
Electronic Journal of Linear Algebra, ISSN 1081-3810

A publication of the International Linear Algebra Society

Volume 36, pp. 519-541, August 2020.

TABLE 1

Lower bounds $\bar{b}_{N}=\operatorname{rank}^{(2)} \overline{\mathrm{J}}_{N}^{(\bar{k})}$ for $p_{N}$; see text.

\begin{tabular}{|c|c|c|c|c|c|c|c|c|c|c|c|c|c|c|}
\hline$N$ & $\overline{\mathrm{J}}_{N}^{(\bar{k})}$ & $\bar{b}_{N}$ & $\bar{k}$ & $N$ & $\overline{\mathrm{J}}_{N}^{(\bar{k})}$ & $\bar{b}_{N}$ & $\bar{k}$ & $N$ & $\overline{\overline{\mathbf{J}}_{N}^{(\bar{k})}}$ & $\bar{b}_{N}$ & $\bar{k}$ & $N$ & $\overline{\mathbf{J}}_{N}^{(\bar{k})}$ & $\bar{b}_{N}$ \\
\hline 1 & $\mathrm{I}_{1}$ & 1 & 1 & 17 & $3_{3} 2_{4}$ & 99 & 2 & 33 & $4_{6} 3_{3}$ & 432 & 2 & 49 & $5_{5} 4_{6}$ & 1008 \\
\hline 2 & $\mathrm{I}_{2}$ & 2 & & 18 & $3_{4} 2_{3}$ & 113 & & 34 & $4_{7} 3_{2}$ & 461 & & 50 & $8_{1} 7_{6}$ & 1053 \\
\hline 3 & $I_{3}$ & 3 & & 19 & $3_{5} 2_{2}$ & 128 & & 35 & $4_{8} 3_{1}$ & 491 & & 60 & $9_{4} 8_{3}$ & 1558 \\
\hline 4 & $\mathrm{I}_{4}$ & 4 & & 20 & $3_{6} 2_{1}$ & 144 & & 36 & 49 & 522 & & 70 & $10_{7}$ & 2163 \\
\hline 5 & $I_{5}$ & 5 & 1 & 21 & $3_{7}$ & 161 & & 37 & $5_{1} 4_{8}$ & 553 & & 80 & $9_{8} 8_{1}$ & 2867 \\
\hline 6 & $2{ }_{1} 1_{4}$ & 7 & 2 & 22 & $4_{1} 3_{6}$ & 178 & & 38 & $5_{2} 4_{7}$ & 585 & & 90 & $10_{9}$ & 3672 \\
\hline 7 & $22_{2} 1_{3}$ & 12 & & 23 & $4_{2} 3_{5}$ & 196 & & 39 & $5_{3} 4_{6}$ & 618 & & 100 & $10_{1} 9_{10}$ & 4572 \\
\hline 8 & $2{ }_{3} 1_{2}$ & 17 & & 24 & $4{ }_{3} 3_{4}$ & 215 & & 40 & $4_{7} 3_{4}$ & 653 & & 1000 & $28_{1} 27_{36}$ & 494240 \\
\hline 9 & $2{ }_{4} 1_{1}$ & 23 & & 25 & $4_{4} 3_{3}$ & 235 & & 41 & $4_{8} 3_{3}$ & 689 & & 2354 & $45_{22} 44_{31}$ & 2755812 \\
\hline 10 & 25 & 30 & & 26 & $4{ }_{5} 3_{2}$ & 256 & & 42 & $4_{9} 3_{2}$ & 726 & & 2355 & $68_{10} 67_{25}$ & 2758160 \\
\hline 11 & $3_{1} 2_{4}$ & 37 & & 27 & 39 & 279 & & 43 & $4_{10} 3_{1}$ & 764 & & $10^{4}$ & $130_{67} 129_{10}$ & 49933229 \\
\hline 12 & $3_{2} 2_{3}$ & 45 & & 28 & $4_{1} 3_{8}$ & 302 & & 44 & $4_{11}$ & 803 & & $10^{5}$ & $441_{120} 440_{107}$ & 4999253405 \\
\hline 13 & $3_{3} 2_{2}$ & 54 & & 29 & $4_{2} 3_{7}$ & 326 & & 45 & $5_{1} 4_{10}$ & 842 & & $10^{6}$ & $1346_{665} 1345_{78}$ & 499991776396 \\
\hline 14 & $3{ }_{4} 2_{1}$ & 64 & & 30 & $4_{3} 3_{6}$ & 351 & & 46 & $5_{2} 49$ & 882 & & 2319293 & $1837_{425} 1836838$ & 2689540338451 \\
\hline 15 & $3_{5}$ & 75 & & 31 & $4_{4} 3_{5}$ & 377 & & 47 & $5_{3} 4_{8}$ & 923 & & 2319294 & $3328_{375} 3327_{322}$ & 2689542657736 \\
\hline 16 & $4_{1} 3_{4}$ & 86 & 2 & 32 & $4_{5} 3_{4}$ & 404 & 2 & 48 & $5_{4} 4_{7}$ & 965 & 2 & $10^{7}$ & $6270_{945} 6269_{650}$ & 49999913368315 \\
\hline
\end{tabular}

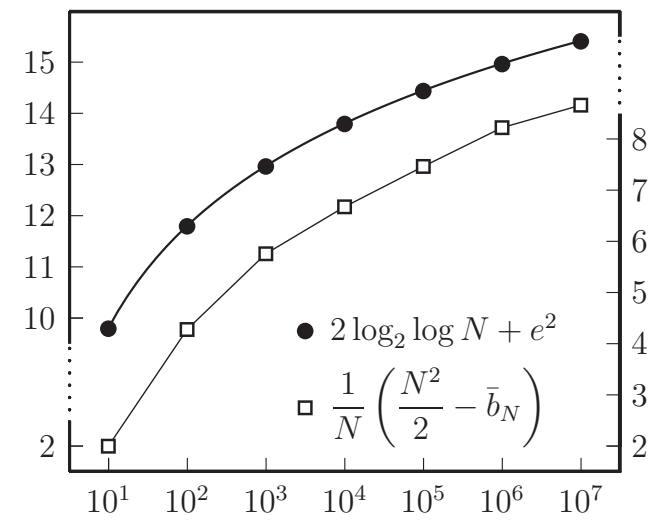

FIGURE 1. Comparison of lower bounds from (7.12), see labelling on the upper left, and $\bar{b}_{N}$ from Table 1 , see labelling on the right, for $N$ in a set of powers of 10 .

The lower bounds derived from optimal matrices $\overline{\mathrm{J}}_{N}^{(\bar{k})}$ turn out to be superior to the previous best lower bounds in [10, Table 2] for $N \in\{10,11\}$ and for $N \geq 48: \operatorname{rank}^{(2)} \circledast \mathrm{U}_{5}\left(\mathrm{I}_{2}, \mathrm{I}_{2}, \mathrm{I}_{2}, \mathrm{I}_{2}, \mathrm{I}_{2}\right)=30$ and $\operatorname{rank}^{(2)} \circledast \mathrm{U}_{5}\left(\mathrm{I}_{3}, \mathrm{I}_{2}, \mathrm{I}_{2}, \mathrm{I}_{2}, \mathrm{I}_{2}\right)=37$ improve the previous lower bounds $p_{10} \geq 28, p_{11} \geq 35$ by 2 in both cases. Note that the witnesses $\bar{J}_{N}^{(\bar{k})}$ for the lower bounds in the present paper always have full rank, while in [10] witnesses for lower bounds where allowed to be rank deficient. 
We finally remark that in the construction used in Proposition 7.3 one could choose different initializations of the set $\left\{\mathrm{J}_{N}^{(1)}: N \in \mathbb{N}\right\}$ with full rank matrices. Choosing $\left\{\mathrm{J}_{N}: N \in \mathbb{N}\right\}$ as in [10, Table 1] instead of $\left\{\mathrm{I}_{N}: N \in \mathbb{N}\right\}$ would result in slightly better lower bounds, but an estimate such as $p_{N}=\frac{N^{2}}{2}+o(N \log \log N)$, if true at all, seems to call for different methods.

Acknowledgements, The author would like to thank an anonymous referee for valuable suggestions that helped to improve the presentation of the paper.

\section{REFERENCES}

[1] F. Ahmed and G. Still. Maximization of homogeneous polynomials over the simplex and the sphere: structure, stability, and generic behavior. J. Optim. Theory Appl., 181:972-996, 2019.

[2] A. Berman, M. Dür, and N. Shaked-Monderer. Open problems in the theory of completely positive and copositive matrices. Electron. J. Linear Algebra, 29:46-58, 2015.

[3] A. Berman and N. Shaked-Monderer. Completely Positive Matrices. World Scientific Publishing Co. Inc., River Edge, NJ, 2003.

[4] I.M. Bomze. On Standard Quadratic Optimization Problems J. Global Optim., 13:369-387, 1998.

[5] I.M. Bomze. Regularity versus degeneracy in dynamics, games, and optimization: a unified approach to different aspects. SIAM Rev., 44:394-414, 2002.

[6] I.M. Bomze. Copositive optimization - recent developments and applications. European J. Oper. Res., 216:509-520, 2012.

[7] I.M. Bomze, P.J.C. Dickinson, and G. Still. The structure of completely positive matrices according to their CP-rank and CP-plus-rank. Linear Algebra Appl., 482:191-206, 2015.

[8] I.M. Bomze, W. Schachinger, and G. Uchida. Think co(mpletely)positive ! - matrix properties, examples and a clustered bibliography on copositive optimization. J. Global Optim., 52:423-445, 2012.

[9] I.M. Bomze, W. Schachinger, and R. Ullrich. From seven to eleven: completely positive matrices with high cp-rank. Linear Algebra Appl., 459:208-221, 2014.

[10] I.M. Bomze, W. Schachinger, and R. Ullrich. New lower bounds and asymptotics for the cp-rank. SIAM J. Matrix Anal. Appl., 36:20-37, 2015.

[11] M. Broom, C. Cannings, and G.T. Vickers. On the number of local maxima of a constrained quadratic form. Proc. R. Soc. Lond. Ser. A, 443:573-584, 1993.

[12] S. Burer. Copositive programming. In: M.F. Anjos and J. B. Lasserre (editors), Handbook of Semidefinite, Cone and Polynomial Optimization: Theory, Algorithms, Software and Applications, Internat. Ser. Oper. Res. Management Sci., Springer, New York, 201-218 2012.

[13] P.J.C. Dickinson and L. Gijben. On the computational complexity of membership problems for the completely positive cone and its dual. Comput. Optim. Appl., 57:403-415, 2014.

[14] H. Dong. Symmetric tensor approximation hierarchies for the completely positive cone. SIAM J. Optim., 23:1850-1866, 2013.

[15] J.H. Drew, C.R. Johnson, and R. Loewy. Completely positive matrices associated with M-matrices. Linear Multilinear Algebra, 37:303-310, 1994.

[16] M. Dür. Copositive programming - a survey. In: M. Diehl, F. Glineur, E. Jarlebring, and W. Michiels (editors), Recent Advances in Optimization and its Applications in Engineering, Springer, Berlin, 3-20, 2010.

[17] J. Fan, J. Nie, and A. Zhou. Completely positive binary tensors. Math. Oper. Res., 44:1087-1100, 2019.

[18] R. Hildebrand. Minimal zeros of copositive matrices. Linear Algebra Appl., 459:154-174, 2014.

[19] R. Hildebrand. Copositive matrices with circulant zero support set. Linear Algebra Appl., 514:1-46, 2017.

[20] S. Liu. Matrix results on the Khatri-Rao and Tracy-Singh products. Linear Algebra Appl., 289:267-277, 1999.

[21] I. Kra and S.R. Simanca. On circulant matrices. Notices Amer. Math. Soc., 59:368-377, 2012.

[22] X. Kuang and L.F. Zuluaga. Completely positive and completely positive semidefinite tensor relaxations for polynomial optimization. J. Global Optim., 70:551-577, 2018.

[23] J. Peña, J.C. Vera, and L.F. Zuluaga. Completely positive reformulations for polynomial optimization. Math. Program., 151:405-431, 2015.

[24] L. Qi. Symmetric nonnegative tensors and copositive tensors. Linear Algebra Appl., 439:228-238, 2013.

[25] L. Qi, C. Xu, and Y. Xu. Nonnegative tensor factorization, completely positive tensors, and a hierarchical elimination algorithm. SIAM J. Matrix Anal. Appl., 35:1227-1241, 2014. 
[26] N. Shaked-Monderer, A. Berman, I.M. Bomze, F. Jarre, and W. Schachinger. New results on the cp rank and related properties of co(mpletely)positive matrices. Linear Multilinear Algebra, 63:384-396, 2015.

[27] N. Shaked-Monderer, I.M. Bomze, F. Jarre, and W. Schachinger. On the cp-rank and minimal cp factorizations of a completely positive matrix. SIAM J. Matrix Anal. Appl., 34:355-368, 2013.

[28] N. Shaked-Monderer. On the DJL conjecture for order 6. Oper. Matrices, 11:71-88, 2017.

[29] A. Zhou and J. Fan. A hierarchy of semidefinite relaxations for completely positive tensor optimization problems. $J$. Global Optim., 75:417-437, 2019. 